\title{
The Optimal Mechanism for Selling to a Budget-Constrained Buyer ${ }^{1}$
}

\author{
Yeon-Koo Che \\ Department of Economics, University of Wisconsin, Madison, Wisconsin 53706 \\ yche@facstaff.wisc.edu \\ and \\ Ian Gale \\ Department of Economics, Georgetown University, Washington, DC 20057 \\ galei@gunet.georgetown.edu
}

Received March 17, 1997; revised December 12, 1999

\begin{abstract}
This paper finds an optimal mechanism for selling a good to a buyer who may be budget-constrained. We consider a seller with one unit of a good facing a buyer with a quasilinear utility function. If the buyer does not face a binding budget constraint, textbook monopoly pricing is optimal. By contrast, the possibility of a binding budget constraint can make it optimal for the seller to use nonlinear pricing, to commit to a declining price sequence, to require the buyer to disclose his budget, or to offer financing. Journal of Economic Literature Classification Numbers: C70, D42, D45, D89, L12. (C) 2000 Academic Press
\end{abstract}

\section{INTRODUCTION}

Sellers often face buyers who differ in their willingness to pay for a good and in their ability to pay. Buyers may face binding budget constraints for a number of reasons. For example, imperfect capital markets may limit a buyer's ability to borrow against future income. The recent sale by the U.S.

\footnotetext{
${ }^{1}$ The authors thank the associate editor and the referee for numerous helpful comments. We have benefitted from discussions with Larry Ausubel, Michael Baye, Ray Deneckere, Prajit Dutta, Faruk Gul, Don Hausch, Mike Peters, Larry Samuelson, Arijit Sen, Chris Snyder, Dan Spulber, Lars Stole, Pete Streufert, and seminar participants at George Washington, Maryland, Northwestern, Toronto, Western Ontario, and Wisconsin. The authors are grateful for support from the NSF (SBR-9423649). Part of the research was conducted while YeonKoo Che visited Yale Law School and Institut d'Anàlisi Econòmica, whose hospitality and financial support are gratefully acknowledged.
} 
Federal Communications Commission of "Personal Communication Services" licenses provided evidence of binding budget constraints (Salant [18]). ${ }^{2}$ Budget constraints are also important in the sale of many consumer durables, which may explain the prevalence of low-interest financing and other similar practices. Despite its practical importance, little attention has been paid to buyers' limited purchasing power or limited liquidity in the literature. ${ }^{3}$

The existing literature considers buyers who are able to pay more than their reservation price for the good. In a typical example, a seller faces a buyer with unit demand who is able to pay more than his reservation price. If the buyer and seller are risk-neutral, posting a single price (i.e., making a take-it-or-leave-it offer) maximizes the seller's expected profit (see Harris and Raviv [6], Riley and Zeckhauser [15], and Stokey [20]). ${ }^{4}$ An implication of this "no haggling" result is that screening consumers through a menu of contracts, committing to a declining intertemporal price sequence, or providing low-interest financing do not benefit the seller. In particular, a declining price sequence could only be explained by the seller's inability to commit to the optimal price (see Gul, Sonnenschein and Wilson [5], for example). Yet, the aforementioned practices are often observed, even when the seller appears to possess commitment power. For instance, Filene's Basement marks down prices according to a posted schedule, based on how long items have been on the shelves.

In this paper we study the optimal method of selling to a buyer whose willingness to pay (his "valuation") and ability to pay (his "budget") are private information. (The results can easily be translated to the case of a continuum of consumers.) The possibility of a binding budget constraint changes the optimal sales mechanism qualitatively. In many settings where a single price would be optimal in the absence of a binding budget constraint, the optimal mechanism here involves a menu of contracts. Put differently, there is nontrivial price discrimination. As such, our problem is not a special

\footnotetext{
${ }^{2}$ Salant [18] notes that "budget parameters" were imposed on some bidders in these auctions. In these and other government auctions, anecdotal evidence suggests that the limited financial resources of small firms are a major concern. For example, the U.S. government has limited the length and size of mineral leases. In timber rights auctions, "setaside sales" have been made available to small firms (see Bergsten et al. [2]). The fact that joint bidding is permitted for small firms in Outer Continental Shelf auctions can also be explained from this perspective (see Hendricks and Porter [7]).

${ }^{3}$ One exception is Sen [19] who considers seller-provided financing as a means of price discrimination when ex ante identical consumers face income fluctuations.

${ }^{4}$ If marginal revenue is non-monotonic and the monopolist has a fixed supply of the good, setting two prices and rationing supply at the lower price may be optimal (see Wilson [21]). There can also be rationing if there is aggregate demand uncertainty, in which case a buyer may get the good with a probability between zero and one (see Wilson [22]). If consumers demand multiple units and have concave utility, the optimal selling mechanism may involve nonlinear pricing (see Maskin and Riley [11], and Mussa and Rosen [14]).
} 
case of the existing models in which buyers are able to pay more than their reservation prices. Moreover, the seller may benefit from imposing a financial disclosure requirement, committing to a price schedule that declines over time, or providing financing.

Two examples illustrate the basic points. The first shows that one cannot analyze our problem by defining the buyer's effective valuation as the minimum of his valuation and budget. Suppose that a seller wishes to sell a good that she values at zero to a risk-neutral buyer. The buyer's valuation is distributed uniformly on $[0,1]$ and his budget, which is an absolute spending limit, is known to equal 2. Since the budget does not bind, the seller is effectively facing the demand curve $p=1-q$. Her optimal strategy is the textbook monopoly solution: make a take-it-or-leave-it offer of $1 / 2$ (Harris and Raviv [6], Riley and Zeckhauser [15], and Stokey [20]). Now suppose that the buyer has a known valuation of 2 , with a budget that is distributed uniformly on $[0,1]$. The minimum of the valuation and budget is again uniform on $[0,1]$, but the budget always binds here. A single take-it-or-leave-it offer is now suboptimal. In fact, it is optimal to require a payment of $2 x$ if the buyer chooses to receive the good with probability $x \in[0,1]$. The different optimal strategies for the seller imply that one cannot analyze our problem simply by looking at the minimum of the valuation and budget (or any other function of the valuation and budget, for that matter).

The next example shows that a cash bond requirement can be used to extract additional revenue. Let the valuation, $v$, be uniformly distributed on $[0,1]$, and let the budget be $w=2-v$. The budget does not bind for any possible $(v, w)$ pair, yet a take-it-or-leave-it offer of $1 / 2$ is now suboptimal. To see this, suppose that the seller charges $2-b$ for the good if the buyer posts a bond of $b$. The buyer clearly has an incentive to post the largest possible bond, so a buyer with budget $w$ will post a bond of $w$ and pay $2-w$. Since $w=2-v$, imposition of a cash bond requirement allows the seller to extract all surplus from the buyer.

These examples show that private information about a buyer's budget is qualitatively different from private information about his valuation. Since a buyer with an unknown budget offers new screening opportunities, seller strategies may emerge here that would not be adopted in the absence of a budget constraint.

The remainder of the paper is organized as follows. In Section 2, we present the basic model. In Section 3, we characterize the optimal mechanism for the "unconditional" case. In that case, the seller cannot use a cash bond requirement to prevent over-reporting of the budget, so prices are not conditioned on the buyer's budget. The optimal mechanism is implemented by a convex, increasing pricing function that relates the probability of receiving the good to the payment. In Section 4 , we turn to the 
"conditional" case. Now, the seller can costlessly prevent the buyer from overstating his budget through the use of a cash bond or financial disclosure requirement, so prices can be conditioned on the buyer's budget. The optimal mechanism is now implemented by a two-dimensional pricing function that is convex and increasing for each possible budget. In that mechanism, types with a budget below a critical level face the same menu of contracts. Types with higher budgets face different menus that offer weakly better deals (i.e., lower payments for given probabilities of receipt). When the valuations and budgets satisfy the monotone likelihood ratio property (MLRP), the optimal mechanism has all budget types facing the same menu of contracts, so the cash bond requirement is superfluous. Section 5 discusses the implications of the availability of financing. Section 6 concludes.

\section{THE MODEL}

A seller has one unit of a good to sell to a buyer. Both parties are risk neutral. A buyer with valuation $v$ puts a value of $v x$ on the "quantity" $x \in[0,1]$. The quantity $x$ can denote the fraction of a unit, if the good is divisible, or the probability that the buyer obtains one unit, if the good is indivisible. (One could also interpret $x$ as the discounted utility associated with obtaining one unit of the good in the future, or as the quality of the good.) The buyer has a budget of $w$, which is the most that he can spend. We say that the buyer's "type" is $(v, w) \in \Theta \equiv[\underline{v}, \bar{v}] \times[\underline{w}, \bar{w}]$. The marginal distribution of $w$ is $G(w)$ and the conditional distribution of $v$ given $w$ is $F(v \mid w)$, with corresponding densities $g(w)$ and $f(v \mid w)$, respectively. The densities are non-zero and continuously differentiable for all $(v, w)$ in the interior of $\Theta$. The seller values the good at zero. ${ }^{5}$

We now present the seller's problem. By the revelation principle, we can restrict attention to direct-revelation mechanisms in which each buyer type has an incentive to report private information truthfully. The mechanism specifies the quantity that the buyer receives, $x(\tilde{v}, \tilde{w}) \in[0,1]$, and the transfer payment he makes, $t(\tilde{v}, \tilde{w}) \in \mathfrak{R}$, if he reports $(\tilde{v}, \tilde{w}) \in \Theta$. Since the seller and buyer are risk neutral, we assume that payments are deterministic. ${ }^{6}$ Formally, a mechanism is a mapping $\langle x, t\rangle: \Theta \rightarrow[0,1] \times(-\infty, \infty)$.

\footnotetext{
${ }^{5}$ Zero is a valid normalization as long as the supports for the buyer's valuation and budget weakly exceed the seller's valuation (i.e., in the "gap" case).

${ }^{6}$ Replacing a random payment with its expected value can only loosen a buyer's budget constraint. At the same time, random payments could conceivably be used to prevent a buyer from exaggerating his budget. The cash bond requirement can accomplish this costlessly, when it is effective. When the cash bond requirement is subject to manipulation by the buyer (e.g., when the buyer can borrow money to exaggerate his wealth), random payments are susceptible to the same problem.
} 
Any feasible mechanism must satisfy certain constraints. First, since the buyer has the option of getting zero surplus by not participating, the following condition must be satisfied:

$$
v x(v, w)-t(v, w) \geqslant 0 \quad \forall(v, w) \in \Theta .
$$

Second, the payment must not exceed the buyer's budget:

$$
t(v, w) \leqslant w \quad \forall(v, w) \in \Theta .
$$

Finally, the buyer must have an incentive to report his information truthfully. We consider two versions of this constraint, depending on the seller's ability to prevent the buyer from overstating his budget. Suppose, first, that the seller can require the buyer to post a bond. In particular, suppose that the seller requires the buyer to post a bond equal to his reported budget (and can thereby prevent him from overstating his budget). In this case, the seller need only prevent higher-budget types from mimicking lower-budget types:

$$
v x(v, w)-t(v, w) \geqslant v x(\tilde{v}, \tilde{w})-t(\tilde{v}, \tilde{w}) \quad \forall(v, w),(\tilde{v}, \tilde{w}) \text { s.t. } \tilde{w} \leqslant w .
$$

A financial disclosure requirement, which induces the buyer to divulge his budget, can have the same effect as the cash bond requirement since the buyer cannot exaggerate his budget, but he can still conceal it. Disclosure requirements are often used in real estate transactions, for example. Such requirements are not used in all transactions, however. One possibility is that the requirement may be ineffective because the buyer can borrow and exaggerate his budget. For this reason, we also consider a stronger version of incentive compatibility:

$$
v x(v, w)-t(v, w) \geqslant v x(\tilde{v}, \tilde{w})-t(\tilde{v}, \tilde{w}) \quad \forall(v, w),(\tilde{v}, \tilde{w}) \text { s.t. } t(\tilde{v}, \tilde{w}) \leqslant w .
$$

This latter constraint requires that each type have no incentive to choose any other contract that it can afford, which may include contracts intended for higher-budget types. Hence, $\left(I C^{\prime}\right)$ is at least as restrictive as $(I C)$. In certain circumstances, however, $\left(I C^{\prime}\right)$ is no more restrictive than $(I C)$, so cash bond and financial disclosure requirements become superfluous.

The seller's problem is to maximize her expected revenue, $\int_{\Theta} t(v, w)$ $f(v, w) d v d w$, subject to these feasibility constraints. Optimal mechanisms with two-dimensional uncertainty are often difficult to characterize (see Armstrong [1], Jehiel, Moldovanu and Stachetti [8], Laffont, Maskin and Rochet [10], McAfee and McMillan [12], and Rochet and Choné [16]). One convenient approach is to focus on the corresponding nonlinear pricing 
problem, which relates quantities to payments. Normally, this would be a onedimensional problem, since no consumer would pay more than another for the same quantity. We show that this is indeed the case for the unconditional mechanisms satisfying $\left(I C^{\prime}\right)$. When the seller can costlessly prevent the buyer from exaggerating his budget, however, she can charge a price that depends on the reported budget. In that case, the problem is two dimensional. In section 4, we solve for the optimal conditional mechanism with $(I C)$ as the incentive compatibility constraint.

\section{OPTIMAL UNCONDITIONAL MECHANISM}

We first find an optimal mechanism that satisfies the stronger version of incentive compatibility (i.e., without an effective financial disclosure or cash bond requirement). Our methodology involves reformulating the mechanism design problem as a nonlinear pricing problem. For any feasible mechanism $\langle x, t\rangle$ satisfying $(I R),(B C)$, and $\left(I C^{\prime}\right)$, there exists a pricing function mapping quantities into payments that generates at least as much revenue as the original mechanism (for each type).

Consider a pricing function with the following properties:

$\tau(0)=0, \tau$ is continuous, increasing, and convex, and $0 \leqslant \tau^{\prime} \leqslant \bar{v}$,

where $\tau^{\prime}$ denotes the highest subgradient (the derivative, if $\tau$ is differentiable). The next lemma allows us to focus on pricing functions satisfying $\left(V F^{\prime}\right)$.

LEMma 1. For any feasible mechanism satisfying $(I R),(B C)$, and $\left(I C^{\prime}\right)$, there exists a pricing function satisfying $\left(V F^{\prime}\right)$ that generates weakly higher expected revenue.

\section{Proof. See Appendix A.}

The pricing function can be given a graphical interpretation. Fix a direct mechanism satisfying $(I R),(B C)$ and $\left(I C^{\prime}\right)$, and plot all of the contracts taken by the wealthiest types: $\langle x(\cdot, \bar{w}), t(\cdot, \bar{w})\rangle$. Now, for each type $(v, \bar{w})$, graph the indifference curve passing through its own contract, and form the upper envelope of these indifference curves. (The indifference curves are straight lines.) Since each type must weakly prefer its own contract, these contracts must all be on the upper envelope, which is increasing and convex. If the upper envelope is offered as a pricing function, each type $(v, \bar{w})$ can be induced to behave exactly as it would under the original mechanism. (If the type- $(\underline{v}, \bar{w})$ buyer got strictly positive surplus, we could increase what each type pays.) The lemma proves that all types with $w<\bar{w}$ 
can also be induced to pay at least as much when facing $\tau$ as they would pay under the original mechanism.

We now express the seller's objective function, for any pricing function. We must first characterize buyer behavior. When facing $\tau$ that satisfies $\left(V F^{\prime}\right)$, a type $(v, w)$ picks $x$ to maximize $v x-\tau(x)$, subject to $\tau(x) \leqslant w$. It is optimal to pick the smallest $x$ such that $v \leqslant \tau^{\prime}(x)$, if $\tau(x)<w$, and to pick $x=\tau^{-1}(w)$ otherwise. Therefore, the types that pick a quantity strictly less than $x$ have $w<\tau(x)$ or $v<\tau^{\prime}(x)$. The measure of those types is

$$
Q(x)=1-\int_{\tau(x)}^{\bar{w}}\left[1-F\left(\tau^{\prime}(x) \mid w\right)\right] g(w) d w .
$$

The seller's revenue is then

$$
\begin{aligned}
\int_{0}^{1} \tau(x) d Q(x)+\tau(1)[1-Q(1)] \\
\quad=\int_{0}^{1} \int_{\tau(x)}^{\bar{w}} \tau^{\prime}(x)\left[1-F\left(\tau^{\prime}(x) \mid w\right)\right] g(w) d w d x,
\end{aligned}
$$

where the equality follows from integration by parts.

Knowledge of the objective function allows us to reformulate the seller's problem as follows:

$$
\begin{aligned}
\max _{\tau} & \int_{0}^{1} \int_{\tau(x)}^{\bar{w}} \tau^{\prime}(x)\left[1-F\left(\tau^{\prime}(x) \mid w\right)\right] g(w) d w d x . \\
& \text { s.t. }\left(V F^{\prime}\right) .
\end{aligned}
$$

We make one assumption before solving this problem. Note first that the expression $v[1-F(v \mid w)]$ represents the expected profit from making a take-it-or-leave-it offer of $v$ to a buyer with budget $w$ (assuming the budget does not bind). Let $H(v \mid w) \equiv 1-F(v \mid w)-v f(v \mid w)$ denote its derivative with respect to $v$. The following assumption, which amounts to an assumption of declining marginal revenue, is satisfied by many well-known distributions such as the uniform distribution.

Assumption 1. For each $w, H(v \mid w)$ is strictly decreasing in $v$.

Let $m^{*} \equiv \arg \max _{p \leqslant \bar{w}} \int_{w}^{\bar{w}} p[1-F(p \mid w)] g(w) d w$. (If $w=\underline{w}=\bar{w}$, let $m^{*}$ $\equiv \arg \max _{p \leqslant w} p[1-F(p\lceil w)]$.) We now show that a seller who knows the budget constraint finds it optimal to make a take-it-or-leave-it offer of $m^{*}$, regardless of whether the constraint binds. 
Proposition 1. It is optimal for the seller to make a take-it-or-leave offer of $m^{*}$, if she knows the buyer's budget.

Proof. Suppose that the budget is known to equal $w$. That is, $w=\underline{w}=$ $\bar{w}$. Suppressing the argument $w$ in $F(\cdot \mid w)$, the seller's problem can be written as:

$$
\max _{\tau} \int_{0}^{1} \tau^{\prime}(x)\left[1-F\left(\tau^{\prime}(x)\right)\right] d x
$$

s.t. $\tau(0)=0, \tau(1) \leqslant w, \tau$ is continuous, increasing, and convex.

(Note that we have ignored the condition on $\tau^{\prime}$ that is in $\left(V F^{\prime}\right)$, but this condition is satisfied by the solution below. The new condition, $\tau(1) \leqslant w$, has no impact on the seller's expected revenue, given zero marginal cost, but it simplifies the exposition.)

The Hamiltonian for the unconstrained version of this problem (i.e., without imposing convexity of $\tau$ ) is

$$
J(x, \tau, u) \equiv u(x)[1-F(u(x))]+\lambda(x) u(x),
$$

where $u$ is the control variable and $\lambda$ is the costate variable. The Hamiltonian necessary conditions are:

$$
\begin{aligned}
\frac{\partial J}{\partial u} & =1-F(u)-u f(u)+\lambda=0, \\
\tau^{\prime} & =u, \\
\lambda^{\prime} & =-\frac{\partial[u(1-F(u))]}{\partial \tau}=0 .
\end{aligned}
$$

Since $\lambda$ is constant, it follows from Assumption 1 that $u$ is constant and unique, so $\tau^{\prime}$ is constant. The transversality conditions are $\lambda(1) \leqslant 0$ and $\lambda(1)[\tau(1)-w]=0$.

The solution takes one of two forms. If $\lambda(1)=0$, then $1-F(u)-u f(u)=$ 0 , so $u(x)$ is the textbook monopoly price for all $x$. This case requires $\tau(1)$ $=m^{*} \leqslant w$. If $\lambda(1)<0$ instead, we have $\tau(1)=w$, implying that $\tau^{\prime}(x)=$ $\tau(1)=w$, since $\tau^{\prime}$ is constant. (Since $1-F(u(1))-u(1) f(u(1))<0$, we have $m^{*}=w$, so $\tau^{\prime}=m^{*}$.) Either way, it is optimal to make a take-it-or-leave-it offer of $m^{*}$.

Proposition 1 shows that there is no price discrimination when the budget is known. An implication is that uncertainty about the budget is necessary to generate price discrimination. We now present the existence result for the general problem $[\mathrm{RS}]$ with $\bar{w}>\underline{w}$. 
THEOREM 1. An optimal unconditional mechanism exists, and it is implemented by a pricing function that satisfies $\left(V F^{\prime}\right)$ and

$$
-\tau^{\prime \prime}(x)\left\{\int_{\tau(x)}^{\bar{w}} \frac{\partial H\left(\tau^{\prime}(x) \mid w\right)}{\partial \tau^{\prime}(x)} g(w) d w\right\}=\left(\tau^{\prime}(x)\right)^{2} f\left(\tau^{\prime}(x) \mid \tau(x)\right) g(\tau(x)) .
$$

if $\tau^{\prime}(x)>\underline{v}$ and $\tau(1)<\bar{w}$.

Proof. The proof is omitted, as it follows the proof of Theorem 2, which is presented in Appendix D.

Given Theorem 1, existence of an optimal direct revelation mechanism follows from Lemma 1, combined with the revelation principle. The revelation principle implies that if there exists an achievable (equilibrium) outcome under any feasible mechanism, it can be implemented by a direct-revelation mechanism. In fact, one can simply choose the set of contracts taken under the optimal pricing function, and use them in the direct-revelation mechanism. No other direct-revelation mechanism yields higher expected revenue, given Lemma 1.

Some properties of the optimal selling mechanism follow directly from (1).

Proposition 2. Suppose that $\bar{w}>\underline{w}$. Then, the optimal pricing function has a strictly convex portion if and only if $m^{*}>\underline{w}$.

Proof. We begin with the "if" part of the statement. Suppose, to the contrary, that the pricing function is linear even though $m^{*}>\underline{w}$. Linearity of $\tau$ implies that the left-hand side of (1) is zero, so we must have $\tau(1) \leqslant \underline{w}$. The program [RS] now reduces to a point-wise maximization problem, which is solved by $\tau^{\prime}(x)=m^{*}$ for all $x$. By linearity and $\left(V F^{\prime}\right)$, however, $\tau(1)=\tau^{\prime}(x)=m^{*}>\underline{w}$, which contradicts the earlier assertion. We conclude that the optimal pricing function has a convex portion if $m^{*}>\underline{w}$.

To prove the "only if" part, suppose that $m^{*} \leqslant \underline{w}$. Setting a single price equal to $m^{*}$ generates an expected profit of $\int_{w}^{\bar{w}} m^{*}\left[1-F\left(m^{*} \mid w\right)\right] g(w) d w$. This is as large as the expected profit from any other pricing function $\tau$ since

$$
\begin{aligned}
\int_{0}^{1} \int_{\underline{w}}^{\bar{w}} m^{*}\left[1-F\left(m^{*} \mid w\right)\right] g(w) d w d x \\
\quad \geqslant \int_{0}^{1} \int_{\tau(x)}^{\bar{w}} \tau^{\prime}(x)\left[1-F\left(\tau^{\prime}(x) \mid w\right)\right] g(w) d w d x,
\end{aligned}
$$

where the left-hand side equals $\int_{\underline{w}}^{\bar{w}} m^{*}\left[1-F\left(m^{*} \mid w\right)\right] g(w) d w$, the righthand side represents the expected profit from the pricing function $\tau$, and the inequality comes from the definition of $m^{*}$. 
Proposition 2 implies that the optimal mechanism involves non-trivial price discrimination when $m^{*}>\underline{w}$. To see this, fix $x \in(0,1)$. The quantity $x$ is chosen by types whose valuation equals the marginal price and whose budget exceeds the required outlay (i.e., $v=\tau^{\prime}(x)$ and $w \geqslant \tau(x)$ ). It is also chosen by types whose valuation exceeds the marginal price and whose budget equals the required outlay (i.e., $v \geqslant \tau^{\prime}(x)$ and $w=\tau(x)$ ). These unconstrained and constrained types, respectively, all purchase $x$. In particular, since $\tau^{\prime}(1)=m$ and $\tau$ is convex, the buyer will choose a quantity in $(0,1)$ whenever $v>m \in(\underline{v}, \bar{v})$ and $w<\tau(1)$. It follows that the set of types choosing quantities in $(0,1)$ has strictly positive measure.

The following two examples illustrate the optimal mechanism and the associated buyer behavior.

EXAmPle 1. Uniform distribution and the Leontief property. Suppose that $(v, w)$ is uniformly distributed on $[0,1]^{2}$. The optimal mechanism is implemented by a nonlinear pricing function that satisfies (1). In this case, (1) simplifies to $2 \tau^{\prime \prime}(x)[1-\tau(x)]-\tau^{\prime}(x)^{2}=0$, which is solved by

$$
\tau^{*}(x)=1-0.143[18.49-10.49 x]^{2 / 3} .
$$

The associated optimal (direct) mechanism is a menu of contracts given by

$$
x(v, w)=\left\{\begin{array}{l}
1 \quad \text { if } \quad v \geqslant 1 / 2, \quad w \geqslant 0.4279 \\
1.7628-0.953 / v^{3} \\
\text { if } 0.3782 \leqslant v<1 / 2, w \geqslant 1-0.143 / v^{2}, \\
1.7628-1.7616(2-w)^{3 / 2} \\
\text { if } v \geqslant 0.3782, w<1-0.143 / v^{2} \\
0 \quad \text { otherwise, }
\end{array}\right.
$$

and $\tau(v, w)=\tau^{*}(x(v, w))$. Figure 1 depicts this menu in terms of the optimal quantity choices. The iso-contract loci, which join types choosing the same contract, have the Leontief shape. That is, the valuation and the budget serve as perfect complements since a buyer whose type is at the kink on an isocontract locus receives a higher quantity only if his valuation and budget both rise.

The following discrete example illustrates additional properties.

EXAmple 2 . The $2 \times 2$ type case and the optimality of haggling. Suppose that the buyer's valuation is either high $\left(v_{2}\right)$ or low $\left(v_{1}\right)$, and his budget is either high $\left(w_{2}\right)$ or low $\left(w_{1}\right)$. In particular, $0<w_{1}<v_{2} \leqslant w_{2}$, and $v_{1}<v_{2}$. Also, suppose that each type $\left(v_{i}, w_{j}\right)$ is realized with probability $\pi_{i j}=1 / 4$. In this case, Lemma 1 implies that an optimal pricing function is piecewise 


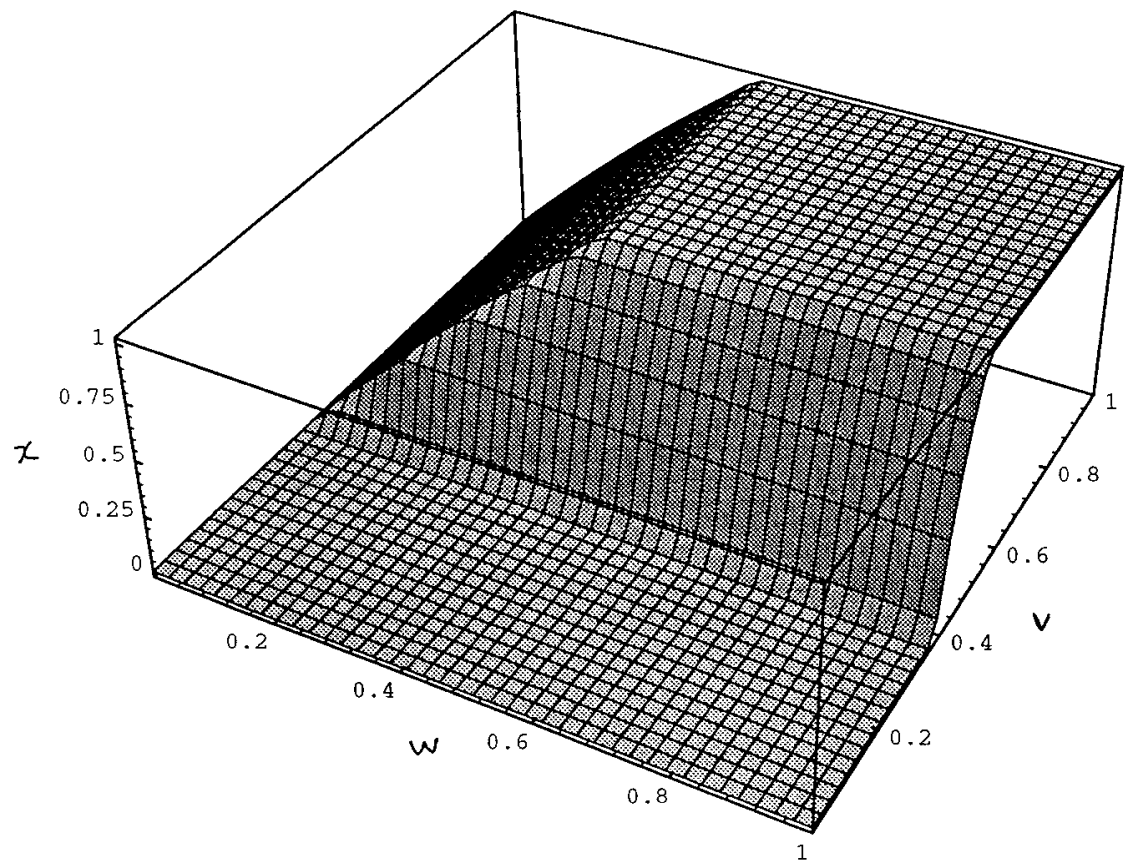

FIG. 1. Quantity choices induced by the optimal pricing function.

linear, with a slope of $v_{1}$ for $x \leqslant \hat{x}$ and $v_{2}$ for $x \geqslant \hat{x}$, for some $\hat{x} \in[0,1]$. In particular, the optimal pricing function is linear with slope $v_{1}$ if $v_{2} / v_{1}<2$, and with slope $v_{2}$ if $v_{2} / v_{1}>3$. When $v_{2} / v_{1} \in(2,3)$, however, the pricing function is strictly convex, with a kink at $\hat{x}=\min \left\{\frac{w_{1}}{v_{1}}, \frac{v_{2}-w_{1}}{v_{2}-v_{1}}\right\}$.

For instance, let $v_{2}=5, v_{1}=2$, and $w_{1}=4$. The optimal take-it-or-leave-it offer would be 2 or 4 , both of which yield expected revenue of 2 . By contrast, the optimal unconditional mechanism is implemented by a pricing function with a kink at $\hat{x}=1 / 3$. When facing this pricing function, the lowvaluation types choose $x=1 / 3$ and pay $t=2 / 3$, and the high-valuation types choose $x=1$ and pay $t=4$ (regardless of the budget), which yields expected revenue of $2 \frac{1}{3}>2$. At first glance, the budget constraint appears to play no role here, as both contracts are affordable for low-budget types, and price discrimination is based on valuations. Yet, the possibility that the seller may face a high-valuation, low-budget type causes her to charge at most 4 and, more importantly, allows her to offer a positive quantity to the low-valuation type. If the buyer were unconstrained for sure (i.e., $w_{1} \geqslant 5$ ), the seller would simply offer $x=1$ at $t=5$, and no contract to the lowvaluation types.

This example also has implications for bargaining. The optimal mechanism can be implemented by committing to a price schedule that declines over time. 
Suppose that the instantaneous discount rate for both the buyer and seller is $r>0$. Let $t_{1}$ satisfy $e^{-r t_{1}}=1 / 3$. Then, the optimal mechanism can be implemented by the price schedule $p(\cdot)$, where $p(t)$ is the price offered at time $t \geqslant 0$ :

$$
p(t)=\left\{\begin{array}{lll}
4 & \text { if } t \leqslant t_{1} \\
2, & \text { if } \quad t>t_{1}
\end{array}\right.
$$

When facing this price sequence, the buyer will purchase immediately if he has the high valuation. Otherwise, he will wait until the price drops to 2, at which point he will purchase one unit. The latter purchase is equivalent to purchasing $1 / 3$ of a unit at time zero at a price of $2 / 3$, which is what the low-valuation type is induced to do in the optimal mechanism above. Such intertemporal price discrimination may be valuable especially when the good is indivisible and lotteries are not feasible. Recall that such a declining price schedule is not optimal absent the possibility of a binding budget constraint. $^{7}$

\section{OPTIMAL CONDITIONAL MECHANISM}

The previous section characterized an optimal selling mechanism for a seller who finds it too costly to prevent over-reporting of the budget. We now characterize the optimal mechanism for a seller who can costlessly prevent over-reporting. The appropriate incentive compatibility condition is now $(I C)$. The remaining constraints are unchanged.

For any feasible mechanism $\langle x, t\rangle$ satisfying $(I R),(B C)$, and $(I C)$, we show that there exists a pricing function that implements the mechanism. In the previous section, such a pricing function was one dimensional since no type would pay more than another for a given quantity. When the seller can costlessly prevent over-reporting, she can charge different types different amounts for a given quantity, so the pricing function is now two dimensional. Consider $T:[0,1] \times[\underline{w}, \bar{w}] \rightarrow \mathfrak{R}_{+}$, where $T(\cdot, w)$ is the pricing function that a buyer faces when he posts a cash bond of $w$. In particular, consider a class of such functions satisfying:

$$
\begin{gathered}
T(0, w)=0, T(\cdot, w) \text { is continuous and convex, } \\
\text { and } 0 \leqslant T_{1}(\cdot, w) \leqslant \bar{v} \quad \forall w \in[\underline{w}, \bar{w}], \\
T(x, \cdot) \text { is nonincreasing } \forall x \in[0,1],
\end{gathered}
$$

${ }^{7}$ As mentioned in the Introduction, a declining price sequence is often attributed to the seller's inability to commit to her optimal price. If the seller has no commitment power in this example, she will have no bargaining power, so she will quote a price of 2 arbitrarily quickly. 
where $T_{1}$ denotes the highest partial subgradient with respect to the first argument.

LEMma 2. For any mechanism $\langle\tilde{x}, \tilde{t}\rangle$ satisfying $(I R),(B C)$, and $(I C)$ there exists $T:[0,1] \times[\underline{w}, \bar{w}] \rightarrow \Re_{+}$satisfying $(V F)$ and $(W F)$ that generates weakly higher expected revenue.

\section{Proof. See Appendix A.}

Consider any $T$ satisfying $(V F)$ and $(W F)$. When facing $T(\cdot, w)$, types with $v \geqslant T_{1}(x, w)$ pick a quantity greater than or equal to $x$, provided $T(x, w) \leqslant w$. Therefore, the probability that a consumer with $w$ will pick a quantity strictly less than $x$ is

$$
Q(x, w)=1-I_{\{T(x, w) \leqslant w\}}\left[1-F\left(T_{1}(x, w) \mid w\right)\right],
$$

where $I_{\{T(x, w) \leqslant w\}}$ is an indicator function taking the value one when $T(x, w) \leqslant w$ and zero otherwise. Integrating by parts, the seller's expected revenue from types with budget $w$ is

$$
\begin{aligned}
\int_{0}^{1} T(x, w) d Q(x, w)+T(1, w)[1-Q(1, w)] \\
\quad=\int_{\{x \in[0,1]: T(x, w) \leqslant w\}} T_{1}(x, w)\left[1-F\left(T_{1}(x, w) \mid w\right)\right] d x .
\end{aligned}
$$

The seller's problem can be written as follows:

$$
\begin{aligned}
& \max _{T} \int_{\underline{w}}^{\bar{w}} \int_{\{x \in[0,1]: T(x, w) \leqslant w\}} T_{1}(x, w)\left[1-F\left(T_{1}(x, w) \mid w\right)\right] d x g(w) d w \\
& \text { s.t. }(V F) \text { and }(W F) .
\end{aligned}
$$

The analysis is simplified by the introduction of a slightly stronger condition than $(W F)$.

For any $w>w^{\prime}, \quad T_{1}(x, w) \leqslant T_{1}\left(x^{\prime}, w^{\prime}\right) \quad$ whenever $\quad T(x, w) \leqslant T\left(x^{\prime}, w^{\prime}\right)$.

The next lemma shows that we can replace $(W F)$ with $\left(W F^{\prime}\right)$.

Lemma 3. When ( $V F)$ holds, $\left(W F^{\prime}\right)$ implies $(W F)$. In addition, given Assumption 1, for any $T$ satisfying $(V F)$ and $(W F)$, there exists $T^{*}$ satisfying $(V F)$ and $\left(W F^{\prime}\right)$ that yields weakly higher expected revenue.

Proof. See Appendix B. 
Given this result, the next lemma shows that the optimal pricing function has a simple structure. Specifically, the optimal pricing function satisfying $(V F)$ and $\left(W F^{\prime}\right)$ is constructed from a convex, one-dimensional function, denoted $\tau$, and a family of affine functions. The importance of this result is that we need only search for this one-dimensional function now.

The proof refers to "ironed-out monopoly prices." Fixing $z \in[0, \bar{w}]$, the ironed-out monopoly prices for types with $w \geqslant z$ are given by the function $\bar{m}_{z}:[z \vee \underline{w}, \bar{w}] \rightarrow \Re$ that solves

$$
\max _{p} \int_{z}^{\bar{w}} p(\tilde{w})[1-F(p(\tilde{w}) \mid \tilde{w})] g(\tilde{w}) d \tilde{w}
$$

s.t. $p:[z \vee \underline{w}, \bar{w}] \rightarrow \mathfrak{R}$ is nonincreasing.

In words, the ironed-out monopoly prices for types with $w \in\left[z, z^{\prime}\right]$ are the optimal take-it-or-leave-it prices for budgets in that interval, given that prices charged to higher-budget types must be no greater than those charged to lower-budget types. ${ }^{8}$ It is also useful to let

$$
m(w) \equiv \underset{p}{\arg \max } p[1-F(p \mid w)]
$$

denote the monopoly price against budget $w$. We now state the lemma.

LEMma 4. For any $T$ satisfying $(V F)$ and $\left(W F^{\prime}\right)$, there exists $T^{*}$ satisfying $(V F)$ and $\left(W F^{\prime}\right)$ that yields weakly higher expected revenue, where $T^{*}(x, w)=$ $\tau(x)$ for $w \leqslant \tau(1)$, and $T^{*}(x, w)=\int_{0}^{x}\left\{\tau^{\prime}(s) \wedge \bar{m}_{\tau(1)}(w)\right\} d s$ for $w>\tau(1)$, for some $\tau$ satisfying $\left(V F^{\prime}\right)$.

\section{Proof. See Appendix C.}

All types with a budget $w \leqslant \tau(1)$ are offered the same deals. Those with budgets above $\tau(1)$ are offered weakly better deals. We refer to $T(\cdot, w)$ as the "pricing function at $w$." Lemma 4 implies that there exists a function $\tau$ satisfying $\left(V F^{\prime}\right)$ such that the optimal pricing function at $w$ is composed of $\tau$ and, possibly, a linear extension whose slope equals the ironed-out monopoly price (see Fig. 2). In particular, the slope of the optimal pricing function at $w$ is equal to the smaller of $\tau^{\prime}$ and the ironed-out monopoly price for that $w$. If the former exceeds the latter for all $x$, the optimal pricing function at $w$ entails charging the ironed-out monopoly price.

\footnotetext{
${ }^{8}$ We ignore the possibility that the budget constraint might bind when defining these prices. This is done for ease of exposition only.
} 
Payment

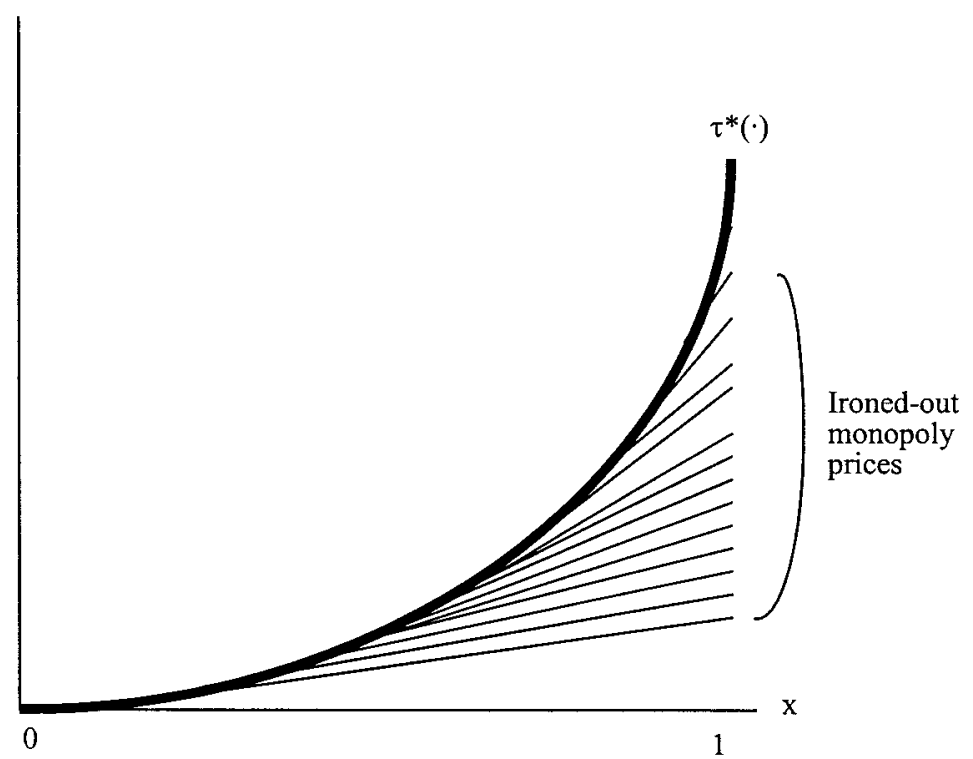

FIG. 2. A family of nonlinear pricing functions.

Using Lemma 4, we can restate the seller's problem as:

$\max _{\tau} \int_{0}^{1}\left\{\int_{\tau(x)}^{\tau(1)} \tau^{\prime}(x)\left[1-F\left(\tau^{\prime}(x) \mid w\right)\right] g(w) d w\right.$

$$
\left.+\int_{\tau(1)}^{\bar{w}}\left(\tau^{\prime}(x) \wedge \bar{m}_{\tau(1)}(w)\right)\left[1-F\left(\tau^{\prime}(x) \wedge \bar{m}_{\tau(1)}(w) \mid w\right)\right] g(w) d w\right\} d x
$$

s.t. $\tau:[0,1] \rightarrow \mathfrak{R}_{+}$is a continuous, convex function with $\tau(0)=0$,

$$
\text { and } 0 \leqslant \tau^{\prime} \leqslant \bar{v} \text {. }
$$

Let $\mu(z) \equiv \bar{m}_{z}(z)$ denote the ironed-out monopoly price for a type with budget $z$ when the seller faces a buyer with budget $z$ or above. Now let $\hat{w}(k) \equiv \inf \left\{w \in[\tau(1), \bar{w}] \mid \bar{m}_{\tau(1)}(w)<k\right\}$ if the set is nonempty; otherwise, let $\hat{w}(k) \equiv \bar{w}$. The existence result for problem [S] follows.

THEOREM 2. There exists an optimal mechanism. It can be implemented by a pricing function $T$ satisfying $T(x, w)=\tau(x)$ for $w \leqslant \tau(1)$, and $T(x, w)=$ $\int_{0}^{x}\left\{\tau^{\prime}(s) \wedge \bar{m}_{\tau(1)}(w)\right\} d s$ for $w>\tau(1)$, where $\tau$ satisfies

$$
-\tau^{\prime \prime}(x)\left\{\int_{\tau(x)}^{\hat{w}\left(\tau^{\prime}(x)\right)} \frac{\partial H\left(\tau^{\prime}(x) \mid w\right)}{\partial \tau^{\prime}(x)} g(w) d w\right\}=\left(\tau^{\prime}(x)\right)^{2} f\left(\tau^{\prime}(x) \mid \tau(x)\right) g(\tau(x))
$$


when $\tau^{\prime}>\underline{v}$ and $\tau(1)<\bar{w}$, with $\tau^{\prime}(1)=\mu(\tau(1))$ if $\tau(1) \in[\underline{w}, \bar{w})$ and $\tau^{\prime}(1) \geqslant \mu(\underline{w})$ if $\tau(1)<\underline{w}$.

\section{Proof. See Appendix D.}

We now turn our attention to the choices made by the buyer. Henceforth, let $T^{*}$ denote an optimal pricing function and let $\tau^{*}$ denote a solution to (2). A type- $(v, w)$ buyer chooses a quantity

$$
x(v, w) \in \underset{x}{\arg \max }\left\{v x-T^{*}(x, w) \text { s.t. } T^{*}(x, w) \leqslant w\right\} .
$$

These optimal choices can be characterized precisely in several cases. Suppose that the ironed-out monopoly price for the lowest budget is weakly below the lowest budget (i.e., $\mu(\underline{w})=\bar{m}_{w}(\underline{w}) \leqslant \underline{w}$ ). It is then optimal to offer the ironed-out monopoly prices, since the budget constraint does not bind for any $w$. That is, the best the seller can do is to offer the ironedout monopoly prices, so a buyer with budget $w$ can receive one unit of the good by paying $\bar{m}_{\underline{w}}(w)$.

Proposition 3. Suppose that $\mu(\underline{w}) \leqslant \underline{w}$. Then, the pricing function $T^{*}(x, w)=\bar{m}_{\underline{w}}(w) x$ for all $(x, w)$ is optimal. The buyer chooses $x(v, w)=1$ if $v \geqslant \bar{m}_{\underline{w}}(w)$, and $x(v, w)=0$ otherwise.

Proof. By definition of $\bar{m}_{w}$, the suggested pricing function solves [S]. In particular, $T^{*}(\cdot, w) \leqslant w$ for all $w$ since $T^{*}(\cdot, w) \leqslant T^{*}(1, w)=\bar{m}_{\underline{w}}(w) \leqslant$ $\mu(\underline{w}) \leqslant \underline{w}$. The buyer behavior is immediate since the budget constraint does not bind.

Although the constraint does not bind when $\mu(\underline{w}) \leqslant \underline{w}$, the seller can use a cash bond requirement to price-discriminate by charging higher prices to types with lower budgets. We next consider the more interesting case in which some types face a binding budget constraint. As in the case of unconditional mechanisms, the seller offers non-trivial price discrimination in the sense that the buyer chooses a quantity $x \in(0,1)$ with positive probability.

Proposition 4. Suppose that $\mu(\underline{w})>\underline{w} \geqslant \underline{v}$. Then, the optimal pricing function at $w=\tau^{*}(1)$ has a strictly convex portion, and there is positive probability that the buyer chooses a quantity $x \in(0,1)$.

Proof. Equation (2) implies that $\tau^{*}$ is convex when $\tau^{*}>\underline{w}$ and $\tau^{*^{\prime}}(x)>\underline{v}$. Thus, for the first statement, it suffices to show that $\tau^{*}(1)>\underline{w}$ and $\tau^{*^{\prime}}(1)>\underline{v}$. Suppose, to the contrary, that $\tau^{*}(1) \leqslant \underline{w}$. Then, by (2), $\tau^{*}$ is linear, so $\tau^{*}(1)=$ $\tau^{* \prime}(1)$. By Theorem $2, \tau^{*^{\prime}}(1) \geqslant \mu(\underline{w})$. But, $\mu(\underline{w})>\underline{w}$, by assumption, implying $\tau^{*}(1)>\underline{w}$, which contradicts $\tau^{*}(1) \leqslant \underline{w}$. 
Now suppose that $\tau^{*}(1)>\underline{w}$ but $\tau^{* \prime}(1) \leqslant \underline{v}$. Convexity gives $\tau^{*}(1)=$ $\int_{0}^{1} \tau^{* \prime}(x) d x \leqslant \int_{0}^{1} \tau^{* \prime}(1) d x \leqslant \underline{v} \leqslant \underline{w}$. This contradicts $\tau^{*}(1)>\underline{w}$. It follows that there is a strictly convex portion.

When facing the optimal pricing function, types with $v>\mu\left(\tau^{*}(1)\right)$ and $w<\tau^{*}(1)$ get $x \in(0,1)$, and pay their entire budgets. (For all such types, we have $v>\tau^{* \prime}(x)$ for $x \in(0,1)$, since Theorem 2 and convexity of $\tau^{*}$ yield $\tau^{* \prime}(x) \leqslant \tau^{* \prime}(1)=\mu\left(\tau^{*}(1)\right)$, but their budgets are below $\tau^{*}(1)$.) The measure of such a set is strictly positive since $\tau^{*}(1)>\underline{w}$ and $\mu<\bar{v}$.

As mentioned in the Introduction, a cash bond or financial disclosure requirement is used for some goods but not for others. This may be because such a requirement does not prevent over-reporting of the budget. Alternatively, it may be because such a requirement is of no value to the seller, even if she can use it effectively. We now explore this latter possibility.

Assumption 2 (Monotone likelihood ratio property). Given $(v, w)$ and $\left(v^{\prime}, w^{\prime}\right)$ in $[\underline{v}, \bar{v}] \times[\underline{w}, \bar{w}]$ such that $w^{\prime} \geqslant w$ and $v^{\prime} \geqslant v$, we have

$$
\frac{f\left(v^{\prime} \mid w^{\prime}\right)}{f\left(v \mid w^{\prime}\right)} \geqslant \frac{f\left(v^{\prime} \mid w\right)}{f(v \mid w)} .
$$

This assumption, together with Assumption 1, implies that $m$ is nondecreasing. ${ }^{9}$ Intuitively, MLRP means that types with higher budgets have higher demand, so the seller would like to charge them more. A cash bond or financial disclosure requirement enables the seller to discriminate against a lower-budget type. Given MLRP, price discrimination against a lowerbudget type is unprofitable, so the financial disclosure requirement loses its value as a device for price discrimination.

Proposition 5. Given Assumption 2, the optimal selling mechanism can be implemented by the pricing function $T^{*}(x, w)=\tau^{*}(x)$ for all $w \in[\underline{w}, \bar{w}]$.

Proof. The statement holds trivially if $\tau^{*}(1) \geqslant \bar{w}$, by Theorem 2, so consider $\tau^{*}(1)<\bar{w}$. In particular, suppose first that $\tau^{*}(1) \in[\underline{w}, \bar{w})$. Then, $\tau^{* \prime}(x) \leqslant \tau^{* \prime}(1)=\mu\left(\tau^{*}(1)\right)=\bar{m}_{\tau^{*}(1)}(w)$ for all $w$, where the inequality holds by convexity, the first equality holds by Theorem 2 , and the second

${ }^{9}$ To see this, note first that a function $f(v, x)$ satisfies the single crossing property in $(v, x)$ if, for any $v>v^{\prime}$ and $x>x^{\prime}$,

$$
f\left(v, x^{\prime}\right) \geqslant(>) f\left(v^{\prime}, x^{\prime}\right) \quad \text { implies } f(v, x) \geqslant(>) f\left(v^{\prime}, x\right) .
$$

Assumptions 1 and 2 imply that $\pi(p, w) \equiv p[1-F(p \mid w)]$ satisfies the single-crossing property in $(p ; w)$ because $\partial^{2} \ln (\pi) / \partial p \partial w \geqslant 0$. Since $\arg \max _{p} \pi(p, w)$ is a singleton, by Assumption 1 , Theorem 4 of Milgrom and Shannon [13] then implies that $m(w)=\operatorname{argmax}_{p} \pi(p, w)$ is nondecreasing in $w$. 
equality holds since $m$ is nondecreasing, given Assumption 2. Theorem 2 then implies that $T^{*}(\cdot, w)=\tau^{*}$ for all $w$.

Now suppose that $\tau^{*}(1)<\underline{w}$. In this case, (2) and Theorem 2 imply that $\tau^{*}$ is linear, with a slope of $\tau^{* \prime}(1) \geqslant \mu(\underline{w})$. Since Assumption 2 implies that $\bar{m}_{w}$ equals some constant $\tilde{m}$, we conclude that the function $T^{*}(x, w)=$ $\tau^{*}(x)=\tilde{m} x$ for all $x$ and $w$ is optimal.

Since $T^{*}(\cdot, w)=\tau^{*}$ for all $w$, the solution to [RS] clearly solves [S]. Hence, the following result holds.

Corollary 1. Given Assumption 2, the optimal unconditional mechanism identified in Theorem 1 is optimal (in the class of conditional mechanisms).

Combining Propositions 1, 2 and 5 yields a generalization of the results of Harris and Raviv, Riley and Zeckhauser, and Stokey.

COROLlaRY 2. It is optimal for the seller to make a take-it-or-leave-it offer at the price $m^{*}$ if she knows the buyer's budget or if $m^{*} \leqslant \underline{w}$ and Assumption 2 holds.

The next example illustrates a case in which a cash bond or financial disclosure requirement is valuable.

EXAmple 3 . The $2 \times 2$ type case and the use of the cash bond requirement. Return to the $2 \times 2$ type case. It turns out that the use of cash bonds increases the seller's expected revenue if and only if

$$
1+\frac{\pi_{11}}{\pi_{21}}<\frac{v_{2}}{v_{1}}<1+\frac{\pi_{12}}{\pi_{22}} \quad \text { and } \quad w_{1}>v_{1} .
$$

The first condition means that the probability of facing a high-valuation type is relatively high (low), conditional on the buyer having a low (high) budget. Thus, the seller would like to charge the high-budget type less than the low-budget type. The second condition makes differential treatment worthwhile for the seller. Given these conditions, the seller will want to charge $v_{1}$ to the high-budget type and $w_{1}\left(>v_{1}\right)$ to the low-budget type. When (3) does not hold, either the seller would want to discriminate against high-budget types, which is not incentive compatible, or such discrimination is unprofitable. If the budget and valuation are independent or positively correlated (which is equivalent to our monotone likelihood ratio property), (3) cannot hold, so the financial disclosure requirement becomes superfluous.

Suppose, for example, that $\pi_{11}=\pi_{22}=1 / 6, \pi_{21}=\pi_{12}=1 / 3, v_{2}=2, v_{1}=1$, and $w_{1}=3 / 2$. The optimal unconditional mechanism is a take-it-or-leave-it offer of 1 , which yields revenue of 1 . In this case, condition (3) holds, so 


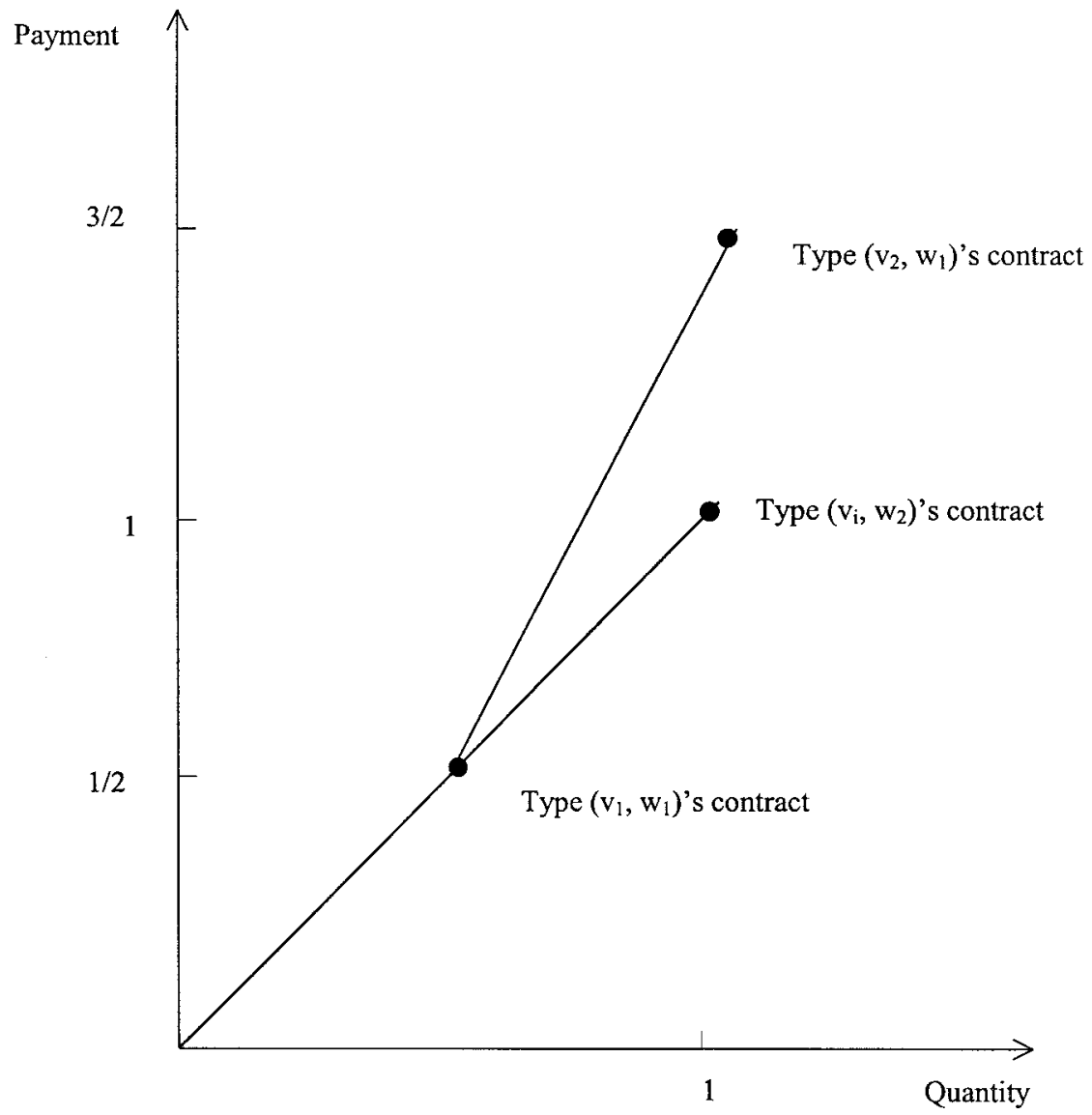

FIG. 3. The use of cash bond requirement.

the cash bond requirement is beneficial. The optimal conditional mechanism charges 1 to a high-budget buyer for $x=1$, and offers a low-budget buyer a choice between a price of $3 / 2$ for $x=1$ and a price of $1 / 2$ for $x=1 / 2$. (The pricing function that implements this outcome is depicted in Fig. 3, which has the shape identified in Lemma 4.) A low-budget type will pick $x=1$ if $v_{i}=2$, and $x=1 / 2$ if $v_{i}=1$. The seller receives expected revenue of $1 \frac{1}{12}$, which exceeds the expected revenue from the take-it-or-leave-it offer.

\section{SELLER-PROVIDED FINANCING}

Thus far, we have assumed that the buyer's budget constitutes an absolute spending limit. In practice, sellers and other lenders often provide 
financing to buyers. The availability of financing raises several interesting issues: How does the ability to provide financing affect the seller's ability to price discriminate? In particular, does it make the buyer's liquidity constraint irrelevant? Why do sellers provide financing when other lenders can supply financing, possibly at lower cost? Rather than simply making the liquidity constraint looser, seller-provided financing enables the seller to exploit the liquidity constraint more effectively as a price-discrimination device. While a third-party lender (such as a credit card company) can also exploit the liquidity constraint, the seller can coordinate the financing and pricing strategies.

To illustrate these points, consider a simple two-period extension of the $2 \times 2$ type case introduced in examples 2 and 3 : In the first period the buyer faces a budget constraint of $w_{1}$ or $w_{2}$, but now he earns enough income in the second period to pay up to the higher valuation, $v_{2}$. For simplicity, assume that the seller can provide financing at zero cost and that both parties do not discount future payoffs. Suppose, first, that

$$
1+\frac{\pi_{12}}{\pi_{22}} \leqslant 1+\frac{\pi_{11}}{\pi_{21}}<\frac{v_{2}}{v_{1}} \quad \text { and } \quad w_{1}<v_{2} .
$$

(Recall that $\pi_{i j}$ is the probability that the buyer's valuation is $v_{i}$ and the budget is $w_{j}$.) The high-budget (liquid) type is more likely to have a high valuation than is the low-budget (illiquid) type, which means that the monotone likelihood ratio property holds. Moreover, both budget types are relatively likely to have the high valuation. It is optimal in this case for the seller to charge a price of $v_{2}$ for the good and to provide zero interest financing of $v_{2}-w_{1}$ (i.e., provide a loan of $v_{2}-w_{1}$, which must be repaid in the next period without interest). This induces a high-valuation buyer to obtain financing if he has a low budget initially. While the seller earns no direct benefit from offering financing, the financing scheme enables her to extract the entire surplus from a type- $v_{2}$ buyer, regardless of the budget, which she could not do previously.

A more interesting possibility arises when

$$
1+\frac{\pi_{11}}{\pi_{21}}<\frac{v_{2}}{v_{1}}<1+\frac{\pi_{12}}{\pi_{22}} \quad \text { and } \quad w_{1}<v_{1} .
$$

The first condition is the same as in (3). It implies that the low-budget type is more likely to have a higher valuation. In this case, it is optimal for the seller to charge a price $v_{1}$ and to offer financing of $v_{1}-w_{1}$, which carries an interest payment of $v_{2}-v_{1}$. When facing such a scheme, a buyer with budget $w_{2}$ will pay $v_{1}$ without obtaining financing. A type- $\left(v_{2}, w_{1}\right)$ buyer will obtain financing, since $w_{1}<v_{1}$, which means paying $v_{2}$ over the two periods. 
This financing scheme enables the seller to extract the entire surplus from the type- $\left(v_{2}, w_{1}\right)$ buyer, while charging a lower price to the high-budget types. Given the first condition of (5), one can easily verify that this is the optimal selling strategy for the seller. Financing is now used as an active instrument of surplus extraction, while the good itself is priced low to attract the high-budget type, who is more likely to have the low valuation. This idea of extracting surplus through financing charges is consistent with casual observation: Many financing programs offered by electronic appliance stores and furniture stores offer low (or deferred) interest for the first three or six months, but then the rates jump up substantially. Many consumers end up paying these high rates rather than paying off the loan early.

A similar opportunity to extract consumer surplus may be available to a third-party lender such as a credit card company. Suppose that the seller cannot provide financing (i.e., the seller cannot collect any payment from the buyer after the initial transaction). Consider the case satisfying (5) and suppose that a monopoly third-party lender charges interest of $v_{2}-v_{1}$ for a loan of $v_{1}-w_{1}$. If $w_{1}$ or the probability of facing a type- $\left(v_{1}, w_{1}\right)$ buyer is sufficiently small, the optimal response from the seller is to charge all types $v_{1}$ for one unit, which gives the same outcome as above, except that the lender now extracts the entire surplus from the low-budget high-valuation buyer. It must be noted, however, that a seller who is able to provide financing can benefit by coordinating the financing and pricing strategies. In particular, the seller internalizes the benefit from low-rate financing on sales of the good whereas the third-party lender does not. For example, in the first case with (4), zero-interest financing will not be profitable for a third-party lender if there is a small probability of default, but the seller may be willing to take that risk if there is a substantial benefit (i.e., a crosssubsidy) from sales of the good.

\section{CONCLUSION}

This paper has characterized an optimal selling mechanism when a buyer may be budget constrained. The optimal mechanism generally involved non-trivial price discrimination, as different types chose different quantities. Depending upon the context, price discrimination may be tied to quantity or quality. It may take the form of a menu of lotteries or intertemporal price discrimination (if the good is indivisible). ${ }^{10}$ Our results also imply

${ }^{10}$ Deneckere and McAfee [3] document many cases where sellers incurred costs to facilitate the use of nonlinear pricing. If the good is perfectly divisible, the seller can implement the optimal mechanism in Theorem 2 through a nonlinear pricing scheme that exhibits quantity or quality premia. On the implementation of quantity premia, see Katz [9]. 
that financial disclosure requirements or seller-provided financing may benefit a seller, particularly when she faces a buyer whose budget (liquid wealth) is low initially, but whose propensity for consumption is high. Our results and their implications are novel since, absent budget constraints, a seller would optimally make a take-it-or-leave-it offer.

There remain some interesting extensions. Our paper considered the case of unit demand and linear preferences in order to highlight the impact of binding budget constraints. A natural extension is to consider a more general model with utility that is concave in quantity. Such a model would explain how the precise form of price discrimination (e.g., the intensity of quantity discounts) changes with the severity of the financial constraint facing the buyer. Second, one might consider a financial constraint that is not an absolute spending limit. Except for Section 5, this paper has focused on a buyer facing an absolute spending limit. The discussion in Section 5 suggests that a more general model would have implications for seller financing as well as the interaction between the seller's strategy and the financing strategies of third-party financial institutions. Default is another important issue that warrants study in this context.

A final, important extension is to explore the welfare implications of the pricing schemes discussed in this paper. In the presence of binding budget constraints, price discrimination can make it profitable for the seller to serve low-budget buyers who would otherwise not be served. Schemes involving installment payments and royalty payments, which are often used in government auctions, may have a similar effect. Likewise, rotating savings and credit associations can enhance welfare by relaxing the budget constraints of the poor. An all-pay auction has a similar effect, by making the object available in small probability units.

\section{APPENDIX A}

\section{Proof of Lemmas 1 and 2}

Proof of Lemma 1. Fix any mechanism $\langle\tilde{x}, \tilde{t}\rangle$ satisfying $(I R),(B C)$, and $\left(I C^{\prime}\right)$. Now consider a pricing function defined by

$$
\tau(x) \equiv \max _{v^{\prime} \in[\underline{v}, \bar{v}]}\left\{v^{\prime} x-\left[v^{\prime} \tilde{x}\left(v^{\prime}, \bar{w}\right)-\tilde{t}\left(v^{\prime}, \bar{w}\right)\right]\right\}+[\underline{v} \tilde{x}(\underline{v}, \bar{w})-\tilde{t}(\underline{v}, \bar{w})] .
$$

It follows from $\left(I C^{\prime}\right)$ that $v^{\prime} \tilde{x}\left(v^{\prime}, \bar{w}\right)-\tilde{t}\left(v^{\prime}, \bar{w}\right)$ is continuous and nondecreasing in $v^{\prime}$, so the maximum is well defined, and $\tau(0)=0$. The maximand in $\tau(x)$ is continuous in $\left(v^{\prime}, x\right)$, so $\tau$ is continuous, by Berge's maximum theorem. Also, the maximand satisfies the strict single-crossing property in $\left(v^{\prime}, x\right)$, so any selection from the set of maximizers, $v(x)$, is nondecreasing 
in $x$. Given the strict single-crossing property, Theorem 4' of Milgrom and Shannon [13] states that $v \geqslant v^{\prime}$ whenever $v \in \arg \max _{\tilde{v}} f(\tilde{v}, x)$ and $v^{\prime} \in$ $\arg \max _{\tilde{v}} f\left(\tilde{v}, x^{\prime}\right)$. Hence, $\tau$ is convex, and $\tau^{\prime}$ is well defined. By the envelope theorem, $\tau^{\prime}(x) \in v(x) \subset[\underline{v}, \bar{v}]$. We conclude that $\tau$ satisfies $\left(V F^{\prime}\right)$.

It now suffices to show that $\tau$ generates weakly higher revenue than $\langle\tilde{x}, \tilde{t}\rangle$. Consider an arbitrary wealthiest type $(v, \bar{w})$. For any $v^{\prime}$, the maximand of the above expression at $x=\tilde{x}(v, \bar{w})$ satisfies:

$$
\begin{aligned}
& v^{\prime} \tilde{x}(v, \bar{w})-\left[v^{\prime} \tilde{x}\left(v^{\prime}, \bar{w}\right)-\tilde{t}\left(v^{\prime}, \bar{w}\right)\right]+[\underline{v} \tilde{x}(\underline{v}, \bar{w})-\tilde{t}(\underline{v}, \bar{w})] \\
& \quad \leqslant v^{\prime} \tilde{x}(v, \bar{w})-\left[v^{\prime} \tilde{x}(v, \bar{w})-\tilde{t}(v, \bar{w})\right]+[\underline{v} \tilde{x}(\underline{v}, \bar{w})-\tilde{t}(\underline{v}, \bar{w})] \\
& \quad=\tilde{t}(v, \bar{w})+[\underline{v} \tilde{x}(\underline{v}, \bar{w})-\tilde{t}(\underline{v}, \bar{w})],
\end{aligned}
$$

where the inequality comes from $\left(I C^{\prime}\right)$. It follows that $\tau(\tilde{x}(v, \bar{w}))=\tilde{t}(v, \bar{w})+$ $[\underline{v} \tilde{x}(\underline{v}, \bar{w})-\tilde{t}(\underline{v}, \bar{w})]$. In other words, if the buyer chooses $x=\tilde{x}(v, \bar{w})$, he pays $\tilde{t}(v, \bar{w})$ plus the net surplus that accrues to the type $(\underline{v}, \bar{w})$.

By definition of $\tau$,

$$
\begin{aligned}
\tau(x) & \geqslant v x-[v \tilde{x}(v, \bar{w})-\tilde{t}(v, \bar{w})]+[\underline{v} \tilde{x}(\underline{v}, \bar{w})-\tilde{t}(\underline{v}, \bar{w})] \\
\Leftrightarrow v x-\tau(x) & \leqslant v \tilde{x}(v, \bar{w})-\tilde{t}(v, \bar{w})-[\underline{v} \tilde{x}(\underline{v}, \bar{w})-\tilde{t}(\underline{v}, \bar{w})] \\
& =v \tilde{x}(v, \bar{w})-\tau(\tilde{x}(v, \bar{w})),
\end{aligned}
$$

for any $x \in[0,1]$, where the last equality holds since $\tau(\tilde{x}(v, \bar{w}))=\tilde{t}(v, \bar{w})+$ $[\underline{v} \tilde{x}(\underline{v}, \bar{w})-\tilde{t}(\underline{v}, \bar{w})]$, from above. Therefore, when facing $\tau$, the type- $(v, \bar{w})$ buyer weakly prefers $\tilde{x}(v, \bar{w})$ to any other $x \in[0,1]$. Let $x^{*}$ be the highest value of $x$ that this type is willing and able to choose. (If $x^{*}>\tilde{x}(v, \bar{w})$, he must be indifferent to choosing $\tilde{x}(v, \bar{w})$, since taking the latter quantity is weakly preferred and feasible.) Note that

$$
\begin{aligned}
\tau\left(x^{*}\right) & \geqslant \min \{\bar{w}, \tau(\tilde{x}(v, \bar{w}))\} \\
& =\min \{\bar{w}, \tilde{t}(v, \bar{w})+[\underline{v} \tilde{x}(\underline{v}, \bar{w})-\tilde{t}(\underline{v}, \bar{w})]\} \geqslant \tilde{t}(v, \bar{w}),
\end{aligned}
$$

where the first inequality follows from the definition of $x^{*}$, and the second inequality follows since $\underline{v} \tilde{x}(\underline{v}, \bar{w})-\tilde{t}(\underline{v}, \bar{w}) \geqslant 0$ by $(I R)$, and $\tilde{t}(v, \bar{w}) \leqslant \bar{w}$ by $(B C)$. Thus, a wealthiest type can be induced to pay at least as much when facing $\tau$ as he would under $\langle\tilde{x}, \tilde{t}\rangle$.

Now consider any type $(v, w), w<\bar{w}$. Since that type has the same preferences as the type $(v, \bar{w})$, the type $(v, w)$ will (weakly) prefer $x^{*}$ to any other quantity for which $\tau(x) \leqslant w$. Two cases must now be considered to show that the type $(v, w)$ pays $\min \left\{w, \tau\left(x^{*}\right)\right\}$. Suppose, first, that $\tau\left(x^{*}\right)>$ $w$. Since $\tau$ is convex, indifference curves are affine, and the type $(v, w)$ 's preferred quantity is $x^{*}$, the type $(v, w)$ will spend $w$. 
Now suppose that $\tau\left(x^{*}\right) \leqslant w$. In this case, the type $(v, w)$ can be induced to choose $x^{*}$ and pay $\tau\left(x^{*}\right)$. This follows since $(\tilde{x}(v, w), \tilde{t}(v, w))$ is on the same indifference curve as $(\tilde{x}(v, \bar{w}), \tilde{t}(v, \bar{w}))$ and $\left(x^{*}, \tau\left(x^{*}\right)-[\underline{v} \tilde{x}(\underline{v}, \bar{w})-\right.$ $\tilde{t}(\underline{v}, \bar{w})])$. (Incentive compatibility guarantees this for types $(v, \bar{w})$ and $(v, w)$ since each has a sufficient budget to afford the other's contract under $\langle\tilde{x}, \tilde{t}\rangle$.) Thus, the type $(v, w)$ pays $\min \left\{w, \tau\left(x^{*}\right)\right\}$ when facing $\tau$.

To show that a type- $(v, w)$ buyer pays at least as much under $\tau$ as under the original mechanism, it suffices to show that $\tau\left(x^{*}\right) \geqslant \tilde{t}(v, w)$. Suppose, to the contrary, that $\tilde{t}(v, w)>\tau\left(x^{*}\right)$. Then,

$$
\begin{aligned}
v \tilde{x}(v, w)-\tilde{t}(v, w) & =v x^{*}-\tau\left(x^{*}\right)+[\underline{v} \tilde{x}(\underline{v}, \bar{w})-\tilde{t}(\underline{v}, \bar{w})] \\
& >v \tilde{x}(v, w)-\tau(\tilde{x}(v, w))+[\underline{v} \tilde{x}(\underline{v}, \bar{w})-\tilde{t}(\underline{v}, \bar{w})],
\end{aligned}
$$

where the first equality follows from the aforementioned indifference and the inequality follows since $\tilde{x}(v, w)>x^{*}$ (by the first equality and the hypothesis that $\left.\tilde{t}(v, w)>\tau\left(x^{*}\right)\right)$ and since $x^{*}$ is the largest maximizer. It follows that $\tilde{t}(v, w)<\tau(\tilde{x}(v, w))-[\underline{v} \tilde{x}(\underline{v}, \bar{w})-\tilde{t}(\underline{v}, \bar{w})]$. But this last fact implies that some type $\left(v^{\prime}, \bar{w}\right), v^{\prime}>v$, would strictly prefer $(\tilde{x}(v, w), \tilde{t}(v, w))$ to $\left(\tilde{x}\left(v^{\prime}, \bar{w}\right)\right.$, $\left.\tilde{t}\left(v^{\prime}, \bar{w}\right)\right)$, which contradicts $\left(I C^{\prime}\right)$. We conclude that $\tilde{t}(v, w) \leqslant \tau\left(x^{*}\right)$.

Proof of Lemma 2. Fix any mechanism $\langle\tilde{x}, \tilde{t}\rangle$ satisfying $(I R),(B C)$, and $(I C)$. Consider the pricing function $T(x, w) \equiv \max _{v \in[v, \bar{v}]}\{v x-[v \tilde{x}(v, w)-$ $\tilde{t}(v, w)]\}$ for each $w \in[\underline{w}, \bar{w}]$. Following Lemma $1, T$ satisfies the conditions of $(V F)$, with the possible exception of the first one. (We deal with that condition later.) Since $v \tilde{x}(v, w)-\tilde{t}(v, w)$ is nondecreasing in $w$, by $(I C), T$ also satisfies $(W F)$.

We now prove that the pricing function yields weakly higher revenue than the original mechanism $\langle\tilde{x}, \tilde{t}\rangle$. Fix any type $(v, w)$. Following the arguments of Lemma 1, a type $(v, w)$ weakly prefers $(\tilde{x}(v, w), \tilde{t}(v, w))$ to $(x, T(x, w)), \forall x \in[0,1]$. We now show that it weakly prefers $(\tilde{x}(v, w), \tilde{t}(v, w))$ to any contract in $\left(x, T\left(x, w^{\prime}\right)\right), \forall w^{\prime}<w$. (By assumption, it can be prevented from choosing a contract in $\left(x, T\left(x, w^{\prime \prime}\right)\right), \forall w^{\prime \prime}>w$.) Suppose, to the contrary, that the type $(v, w)$ strictly prefers $\left(x, T\left(x, w^{\prime}\right)\right)$ to $(\tilde{x}(v, w), \tilde{t}(v, w))$ for some $x \in[0,1]$ and some $w^{\prime}<w$. As noted above, a type $\left(v, w^{\prime}\right)$ weakly prefers $\left(\tilde{x}\left(v, w^{\prime}\right), \tilde{t}\left(v, w^{\prime}\right)\right)$ to $\left(x, T\left(x, w^{\prime}\right)\right)$. Since a type $(v, w)$ has the same preferences as a type $\left(v, w^{\prime}\right)$, the former type strictly prefers $\left(\tilde{x}\left(v, w^{\prime}\right), \tilde{t}\left(v, w^{\prime}\right)\right)$ to $(\tilde{x}(v, w), \tilde{t}(v, w))$, which contradicts the hypothesis that $\langle\tilde{x}, \tilde{t}\rangle$ satisfies (IC).

Consider the new pricing function $\widetilde{T} \equiv \max \{T, 0\}$. This function satisfies ( $V F)$ and $(W F)$. (Since $\widetilde{T}(0, w)=0$, the first part of $(V F)$ is now satisfied.) The proof is completed by noting that $\widetilde{T}$ induces the same behavior from each type as $T$, except where $\widetilde{T}$ prevents a negative payment. 


\section{APPENDIX B}

\section{Proof of Lemma 3}

We first show that $\left(W F^{\prime}\right)$ implies $(W F)$, when $(V F)$ holds. Suppose that $(V F)$ and $\left(W F^{\prime}\right)$ hold, and yet $T(x, w)>T\left(x, w^{\prime}\right)$ for some $x \in[0,1]$ and $w>w^{\prime}$, thereby violating $(W F)$. Then, $(V F)$ implies that there exists $x^{\prime}<x$ such that $T\left(x^{\prime}, w\right)=T\left(x^{\prime}, w^{\prime}\right)$ and $T_{1}\left(x^{\prime}, w\right)>T_{1}\left(x^{\prime}, w^{\prime}\right)$. This latter fact contradicts $\left(W F^{\prime}\right)$, however. We conclude that $\left(W F^{\prime}\right)$ implies $(W F)$ when $(V F)$ holds.

We now show that, for any $T$ satisfying $(V F)$ and $(W F)$, there exists $T^{*}$ satisfying $(V F)$ and $\left(W F^{\prime}\right)$ that yields (weakly) higher expected revenue.

Fix $T$ satisfying $(V F)$ and $(W F)$. We need the following preliminary step. For any $(v, w)$, let

$$
U(v, w) \equiv \max _{x \in[0,1]}\{v x-T(x, w) \text { s.t. } T(x, w) \leqslant w\}
$$

denote a type- $(v, w)$ buyer's equilibrium utility under $T$, and let $X(v, w)$ denote the largest maximizer. (Given continuity of $T(\cdot, w)$, the maximum is well-defined.) Let $\bar{x}(w) \equiv X(\bar{v}, w)$ and let $\bar{t}(w) \equiv T(X(\bar{v}, w), w)$. Since $\bar{x}$ is the largest maximizer, $T(x, \cdot)$ is nonincreasing, and $T_{1}(\cdot, w) \leqslant \bar{v}$, it follows that $\bar{x}$ is nondecreasing. For each $w \in[\underline{w}, \bar{w}]$, let

$$
y(x, w) \equiv\left\{\begin{array}{lll}
x \frac{\bar{t}(w)}{\bar{x}(w)} & \text { if } \quad x \leqslant \bar{x}(w) \\
\bar{t}(w)+\bar{v}(x-\bar{x}(w)) & \text { if } \quad x>\bar{x}(w) .
\end{array}\right.
$$

Now let

$$
T^{*}(\cdot, w) \equiv \operatorname{conv}\left\{y\left(\cdot, w^{\prime}\right)\right\}_{w^{\prime} \in[\underline{w}, w]}
$$

for each $w$, where the right-hand side denotes the largest convex function (weakly) below the set of functions $\left\{y\left(\cdot, w^{\prime}\right)\right\}_{w^{\prime} \in[w, w]}$. By definition, $T^{*}(0, \cdot)=0, T^{*}(\cdot, w)$ is nondecreasing, continuous and convex for all $w$, with $T_{1}^{*}(x, \cdot) \leqslant \bar{v}$. Hence, $T^{*}$ satisfies $(V F)$.

We next show that $T^{*}$ satisfies $\left(W F^{\prime}\right)$. Fix any $x, x^{\prime}$, and $w>w^{\prime}$ such that $T^{*}(x, w) \leqslant T^{*}\left(x^{\prime}, w^{\prime}\right)$. There exists an interval, $A(x, \varepsilon) \equiv((x-\varepsilon) \vee$ $0,(x+\varepsilon) \wedge 1)$, throughout which $T^{*}(\cdot, w)=T^{*}\left(\cdot, w^{\prime}\right)$ or $T^{*}(\cdot, w)<$ $T^{*}\left(\cdot, w^{\prime}\right)$, for some $\varepsilon>0$. In the former case, $T^{*}(x, w) \leqslant T^{*}\left(x^{\prime}, w^{\prime}\right)$ implies $x \leqslant x^{\prime}$, which in turn implies $T_{1}^{*}\left(x, w^{\prime}\right) \leqslant T_{1}^{*}\left(x^{\prime}, w^{\prime}\right)$, by convexity. At the same time, equality of the functions in a neighborhood of $x$ implies $T_{1}^{*}(x, w)=T_{1}^{*}\left(x, w^{\prime}\right)$. Together, we have $T_{1}^{*}(x, w) \leqslant T_{1}^{*}\left(x^{\prime}, w^{\prime}\right)$, which means that $\left(W F^{\prime}\right)$ is satisfied. 
Now assume that $T^{*}(\cdot, w)<T^{*}\left(\cdot, w^{\prime}\right)$ throughout $A(x, \varepsilon)$. Suppose that $y_{1}\left(x^{\prime}, w^{\prime}\right)=\bar{v}$. Then, $T_{1}\left(x^{\prime}, w^{\prime}\right)=\bar{v}$ ( since $\bar{x}$ is nondecreasing). In this case, the proof is trivial since $T_{1}(\cdot, w)$ cannot exceed $\bar{v}$. Now suppose that $y_{1}\left(x^{\prime}, w^{\prime}\right)<\bar{v}$. This means that $x^{\prime}<\bar{x}\left(w^{\prime}\right)$. Since $\bar{x}$ is nondecreasing, it follows that $x<\bar{x}(\tilde{w})$ for all $\tilde{w}>w^{\prime}$. Hence, $T^{*}(\cdot, w)$ must be on the linear segment from the origin, or on the linear extension of $T^{*}\left(\cdot, w^{\prime}\right)$. Formally, $T^{*}(\cdot, w)$ is on the highest convex function that lies below $T^{*}\left(\cdot, w^{\prime}\right)$ and passes through the origin. Again, $T^{*}(x, w) \leqslant T^{*}\left(x^{\prime}, w^{\prime}\right)$ implies $T_{1}^{*}(x, w) \leqslant$ $T_{1}^{*}\left(x^{\prime}, w^{\prime}\right)$. We conclude that $T^{*}$ satisfies $\left(W F^{\prime}\right)$.

Let

$$
U^{*}(v, w) \equiv \max _{x \in[0,1]}\left\{v x-T^{*}(x, w) \text { s.t. } T^{*}(x, w) \leqslant w\right\}
$$

denote the type- $(v, w)$ buyer's equilibrium expected utility under $T^{*}$, and let $x^{*}(v, w)$ denote the smallest maximizer. Now observe that $T^{*}(\cdot, w) \geqslant$ $T(\cdot, w)$ for all $w$, so $U^{*}(\cdot, w) \leqslant U(\cdot, w)$. Since the graph of $T^{*}(\cdot, w)$ contains the contract $(\bar{x}(w), \bar{t}(w))$, we have $U^{*}(\bar{v}, w)=U(\bar{v}, w)$.

The expected revenue generated by $T^{*}(\cdot, w)$ from types with $w$ is

$$
\begin{aligned}
& \int_{x^{*}(\underline{v}, w)}^{\bar{x}(w)} T_{1}^{*}(x, w)\left[1-F\left(T_{1}^{*}(x, w) \mid w\right)\right] d x+T^{*}\left(x^{*}(\underline{v}, w), w\right) \\
& =\int_{\underline{v}}^{\bar{v}} v[1-F(v \mid w)] d x *(v, w)+T^{*}\left(x^{*}(\underline{v}, w), w\right) \\
& =-\int_{\underline{v}}^{\bar{v}} H(v \mid w) x^{*}(v, w) d v-U^{*}(\underline{v}, w) \\
& =-H(\bar{v} \mid w) U^{*}(\bar{v}, w)+[H(\underline{v} \mid w)-1] U^{*}(\underline{v}, w) \\
& +\int_{\underline{v}}^{\bar{v}} \frac{\partial H(v \mid w)}{\partial v} U^{*}(v, w) d v \\
& \geqslant-H(\bar{v} \mid w) U(\bar{v}, w)+[H(\underline{v}, w)-1] U(\underline{v}, w) \\
& +\int_{\underline{v}}^{\bar{v}} \frac{\partial H(v \mid w)}{\partial v} U(v, w) d v \\
& =\int_{X(\underline{v}, w)}^{X(\bar{v}, w)} T_{1}(x, w)\left[1-F\left(T_{1}(x, w) \mid w\right)\right] d x+T(X(\underline{v}, w), w),
\end{aligned}
$$

where the last line represents the expected revenue under $T$ from types with $w$. The first equality results from a change of variables, since $T_{1}^{*}\left(x^{*}(v, w), w\right)=v$ for almost every $v$ such that $x^{*}(v, w)<\bar{x}(w)$. The second and third equalities are the result of integration by parts, combined with the envelope theorem result that $x^{*}(v, w)=\partial U^{*}(v, w) / \partial v$. The inequality follows since $\partial H(v \mid w) / \partial v$ is 
strictly negative (given Assumption 1 ), $U^{*}(\bar{v}, w)=U(\bar{v}, w)$, and $U^{*}(\cdot, w) \leqslant$ $U(\cdot, w)$. The last equality follows from the first three equalities taken in the reverse order, with $T$ replacing $T^{*}$. Since the revenue ranking holds for all $w, T^{*}$ yields weakly higher expected revenue than $T$.

\section{APPENDIX C}

\section{Proof of Lemma 4}

The proof involves several lemmas. The first shows that we can restrict attention to pricing functions such that all types with budgets below a critical level (the constrained types) are offered the same deals. We subsequently describe the better deals that are offered to those with higher budgets.

Lemma C1. For any $T$ satisfying $(V F)$ and $\left(W F^{\prime}\right)$, there exists $T^{*}$ satisfying $(V F)$ and $\left(W F^{\prime}\right)$ that yields weakly higher expected revenue, where $T^{*}(x, w)=\tau(x)$ for $w \leqslant \tau(1)$, and $T_{1}^{*}(x, w) \leqslant \tau^{\prime}(x)$ for $w \geqslant \tau(1)$, for some $\tau$ satisfying $\left(V F^{\prime}\right)$.

Proof. Fix any $T$ satisfying $(V F)$ and $\left(W F^{\prime}\right)$. Let $w^{*} \equiv \sup \{w \in$ $[\underline{w}, \bar{w}] \mid T(1, w) \geqslant w\}$ if the set is nonempty; otherwise, let $w^{*} \equiv \underline{w}$. Let $\tau(x) \equiv T\left(x, w^{*}\right)$ if $T\left(1, w^{*}\right) \geqslant w^{*} ;$ otherwise, let $\tau(x) \equiv \inf _{w<w^{*}} T(x, w)$. Clearly, $\tau$ satisfies $\left(V F^{\prime}\right)$. Also, since $\left(W F^{\prime}\right)$ implies $(W F), T(x, w) \leqslant \tau(x)$ for all $w>\tau(1)$. Then, $\left(W F^{\prime}\right)$ further implies that $T_{1}(x, w) \leqslant \tau^{\prime}(x)$ for all $w>\tau(1)$.

Consider a new pricing function, $T^{*}$, with $T^{*}(x, w) \equiv \tau(x)$ for $w \leqslant \tau(1)$, and $T^{*}(x, w) \equiv T(x, w) \leqslant \tau(x)$ for $w>\tau(1)$. This new function clearly satisfies $(V F)$ and $\left(W F^{\prime}\right)$, given the way $\tau$ is defined. Under this new function, all buyer types with $w>\tau(1) \equiv w^{*}$ face the same menu as under $T$, so their behavior is unchanged. Now consider a type- $(v, w)$ buyer, $w<\tau(1)$. Suppose that type picks the quantity $x$ when facing $T(\cdot, w)$. This implies that, for any $x^{\prime}<x, v-T_{1}\left(x^{\prime}, w\right) \geqslant 0$. Fix $x^{*} \in[0,1]$ such that $\tau\left(x^{*}\right)=T(x, w)$. The quantity $x^{*}$ is well defined since $\tau(1)>w \geqslant T(x, w)>\tau(0)$, and since $\tau$ is continuous. By $\left(W F^{\prime}\right)$ this implies that, for any $x^{\prime \prime}<x^{*}, \tau^{\prime}\left(x^{\prime \prime}\right) \leqslant T_{1}\left(x^{\prime}, w\right)$ for some $x^{\prime}<x$, so we have $v-\tau^{\prime}\left(x^{\prime \prime}\right) \geqslant 0$. It follows that such a buyer will pick a quantity weakly higher than $x^{*}$, when facing $\tau \equiv T\left(\cdot, w^{*}\right)$, so its payment will be weakly higher than $T(x, w)$, which is its payment under $T$. Since this argument works for any buyer type with $w<\tau(1)$, we conclude that $T^{*}$ yields weakly higher expected revenue than $T$.

We now turn our attention to the unconstrained types. The next main result (contained in Lemma C6) refers to the ironed-out monopoly price 
function, $\bar{m}_{z}$, which is the solution to program $\left[\mathrm{M}_{z}\right]$. We step back to characterize this solution first. Since the integrand of the objective function in $\left[\mathrm{M}_{z}\right]$ is continuous, existence of the solution is easily established (see Theorem 2.1 of Fleming and Rishel [4, p.63]). The solution is unique since, by Assumption 1, the integrand of the objective function is strictly concave in $p$, and the set of feasible functions (i.e., nonincreasing functions) is convex. The unique solution is characterized by the necessary and sufficient conditions below.

Consider the Lagrangean equation associated with the problem:

$$
K\left(w, p, p^{\prime}\right)=p[1-F(p \mid w)]+\alpha p^{\prime}-\beta p^{\prime},
$$

where $\alpha$ is the costate variable and $\beta$ is the multiplier associated with the monotonicity constraint. The solution, $\bar{m}_{z}$, must satisfy the following necessary and sufficient conditions:

$$
\begin{aligned}
\alpha^{\prime}(w) & =-H(p(w) \mid w), \\
\alpha(w)-\beta(w) & =0 \quad \text { and } \quad p^{\prime}(w) \beta(w)=0, \\
\alpha(z \vee \underline{w}) & =\alpha(\bar{w})=0 .
\end{aligned}
$$

The necessary conditions are sufficient since $K$ is concave in $\left(p, p^{\prime}\right)$.

The following lemmas result from inspection of $(\mathrm{C} 1)-(\mathrm{C} 3)$.

LEMMA C2. If $\bar{m}_{z}$ is strictly decreasing in a neighborhood of $w$, then $\bar{m}_{z}(w)$ $=m(w)$. Now suppose that $\bar{m}_{z}$ equals a constant $\tilde{m}$ over an interval, and let $I(\tilde{m}) \equiv\left\{w: \bar{m}_{z}(w)=\tilde{m}\right\}$ be the largest such interval. Then $\int_{I(\tilde{m})} H(\tilde{m} \mid w) d w=0$.

Proof. Suppose that $\bar{m}_{z}$ is strictly decreasing in a neighborhood of $w$. By (C2), $\alpha=\beta=0$ in that neighborhood. By (C1), this implies that $H\left(\bar{m}_{z}(w) \mid w\right)=0$, or $\bar{m}_{z}(w)=m(w)$. Now suppose that $\bar{m}_{z}$ equals a constant $\tilde{m}$ over $I(\tilde{m})$. Then, $\int_{I(\tilde{m})} H(\bar{m} \mid w) d w=\alpha(\sup I(\tilde{m}))-\alpha(\inf I(\tilde{m}))=0$, where $\alpha(\sup I(\tilde{m}))=\alpha(\inf I(\tilde{m}))=0$ since $\bar{m}_{z}$ is strictly decreasing immediately outside the interval $I(\tilde{m})$, by definition.

Lemma C3. Fix $z$ and $z^{\prime}$ such that $z<z^{\prime} \leqslant \bar{w}$. If $\bar{m}_{z}$ is strictly decreasing in a neighborhood of $z^{\prime}$, then $\bar{m}_{z}(w)=\bar{m}_{z^{\prime}}(w)$ for all $w \geqslant z^{\prime}$.

Proof. Clearly, $\bar{m}_{z}$ satisfies (C1) and (C2) for $w \geqslant z^{\prime}$, and $\alpha(\bar{w})=0$, by the free-end condition. Since $\bar{m}_{z}$ is strictly decreasing in a neighborhood of $z^{\prime}$, the proof of Lemma C2 implies that $\alpha\left(z^{\prime}\right)=0$. Therefore, $\bar{m}_{z}$ also satisfies (C3) for the program $\left[\mathrm{M}_{z^{\prime}}\right]$. Since the solution to $\left[\mathrm{M}_{z^{\prime}}\right]$ is unique, and $(\mathrm{C} 1)-(\mathrm{C} 3)$ are necessary and sufficient, we have $\bar{m}_{z}(w)=\bar{m}_{z^{\prime}}(w)$ for all $w \geqslant z^{\prime}$. 
Two more preliminary results are needed before we are ready to prove the result.

Lemma C4. Suppose that $m$ is strictly increasing over the interval $\left[w_{1}, w_{2}\right]$. Then, for any pricing function $\{T(\cdot, w)\}_{w \in\left[w_{1}, w_{2}\right]}$ satisfying $(V F)$ and $\left(W F^{\prime}\right)$, there exists a one-dimensional pricing function, $y:[0,1] \rightarrow R$, such that replacing $T(\cdot, w)$ with $y$ for $w \in\left[w_{1}, w_{2}\right]$ increases expected revenue (while satisfying $(V F)$ and $\left.\left(W F^{\prime}\right)\right)$.

Proof. Let $w(x) \equiv \sup \left\{w \in\left[w_{1}, w_{2}\right] \mid T_{1}(x, w)>m(w)\right\}$. (If $T_{1}(x, w) \leqslant$ $m(w)$ for all $w$ in the interval, let $\left.w(x) \equiv w_{1}\right)$. Now let $\underline{t}(x) \equiv T\left(x, w_{1}\right)$ and $\bar{t}(x) \equiv T\left(x, w_{2}\right)$. Finally, define a pricing function, $y$, such that $y^{\prime}(x) \equiv$ $\left\{m(w(x)) \wedge \underline{t}^{\prime}(x)\right\} \vee \bar{t}^{\prime}(x)$. Since $\bar{t}$ and $\underline{t}$ satisfy $(V F)$, and $w$ is nondecreasing, $y$ also satisfies $(V F)$. Since $\{T(\cdot, w)\}_{w \in\left[w_{1}, w_{2}\right]}$ satisfies $\left(W F^{\prime}\right)$, we have $T_{1}(x, w) \leqslant m(w(x)) \leqslant m(w)$ for $w \in\left(w(x), w_{2}\right]$, where the first inequality follows from the definition of $w$ and the second follows from the hypothesis that $m$ is nondecreasing. By Assumption 1, raising $T_{1}(x, w)$ to $y^{\prime}(x)$ for $w \in\left(w(x), w_{2}\right]$ weakly increases expected revenue. Similarly, we have $T_{1}(x, w) \geqslant m(w(x)) \geqslant m(w)$ for $w \in\left[w_{1}, w(x)\right)$, so lowering $T_{1}(x, w)$ to $y^{\prime}(x)$ in that interval weakly increases expected revenue. The above two inequalities also imply that $y$ satisfies $\left(W F^{\prime}\right)$ with respect to $T(\cdot, w)$ outside that interval.

By Lemma C4, we can replace $\{T(\cdot, w)\}_{w>\tau(1)}$ with a one-dimensional pricing function throughout an interval where $m$ is strictly increasing. In particular, suppose that there are $J \geqslant 0$ non-intersecting, non-abutting intervals, $\left\{\left[w_{1}^{j}, w_{2}^{j}\right]\right\}_{j=1}^{J}$, on which $m$ is strictly increasing. Let $y_{j}$ denote the optimal pricing function for interval $j$, let $y_{0} \equiv \tau$, and let $y_{J+1} \equiv 0$. We now show that we can restrict attention to pricing functions that comprise linear extensions to $\tau$.

Lemma C5. For any $T$ satisfying $(V F)$ and $\left(W F^{\prime}\right)$, there exists $T^{*}$ that yields weakly higher expected revenue and satisfies $(V F)$ and $\left(W F^{\prime}\right)$, where $T^{*}(x, w)=\int_{0}^{x}\left\{\tau^{\prime}(s) \wedge t_{1}(s, w)\right\} d s$ for $w>\tau(1)$, for some linear function $t(\cdot, w)$.

Proof. There are two cases. If $J=0, m$ is nonincreasing. By Assumption 1, it is optimal to set $t_{1}(x, w)=m(w)$ for all $x \in[0,1]$. Now suppose that $J>0$. Consider an interval $\left(w_{2}^{i}, w_{1}^{i+1}\right), 0 \leqslant i \leqslant J-1$. By definition, $m$ is nonincreasing over this interval. For any $w$ in the interval, it is optimal to set $t_{1}(x, w)$ $=\left\{m(w) \wedge y_{i}^{\prime}(x)\right\} \vee y_{i+1}^{\prime}(x)$, for all $x$, by Assumption 1. Since $m$ is nonincreasing in this interval, $T^{*}$ clearly satisfies $(V F)$ and $\left(W F^{\prime}\right)$, if $t_{1}$ is chosen in this way. It now suffices to show that each $y_{i}$ is linear. We can write $y_{i}(x) \equiv \int_{0}^{x}\left[\left\{z_{i}^{\prime}(s) \wedge y_{i-1}^{\prime}(s)\right\} \vee y_{i+1}^{\prime}(s)\right] d s$, for some $z_{i}$. (Since $y_{i}$ must 
satisfy ( $W F^{\prime}$ ), such a $z_{i}$ exists.) If $z_{i}$ is linear for $0<i \leqslant J, y_{i}$ is linear. It remains to show that each $z_{i}$ is linear.

Let $\phi_{i}^{-}\left(z_{i}^{\prime}(x), w\right) \equiv y_{i-1}^{\prime}(x) \wedge\left[m(w) \vee\left\{z_{i}^{\prime}(x) \vee y_{i+1}^{\prime}(x)\right\}\right]$ and let $\phi_{i}^{+}\left(z_{i}^{\prime}(x), w\right) \equiv y_{i+1}^{\prime}(x) \vee\left[m(w) \wedge\left\{z_{i}^{\prime}(x) \wedge y_{i-1}^{\prime}(x)\right\}\right]$. Then, given a choice of $z_{i}$ for the interval $\left[w_{1}^{i}, w_{2}^{i}\right]$, the argument above implies that it is optimal to set $t_{1}(x, w)=\phi_{i}^{-}\left(z_{i}^{\prime}(x), w\right)$ for the interval $\left(w_{2}^{i-1}, w_{1}^{i}\right)$, and to set $t_{1}(x, w)=\phi_{i}^{+}\left(z_{i}^{\prime}(x), w\right)$ for the interval $\left(w_{2}^{i}, w_{1}^{i+1}\right)$. Therefore, $z_{i}$ must solve:

$$
\begin{aligned}
\max _{z_{i}} & \int_{0}^{1}\left\{\int_{w_{2}^{i-1}}^{w_{1}^{i}} \phi_{i}^{-}\left(z_{i}^{\prime}(x), x\right)\left[1-F\left(\phi_{i}^{-}\left(z_{i}^{\prime}(x), w\right) \mid w\right)\right] g(w) d w\right. \\
& +\int_{w_{1}^{i}}^{w_{2}^{i}}\left[\left\{z_{i}^{\prime}(x) \wedge y_{i-1}^{\prime}(x)\right\} \vee y_{i+1}^{\prime}(x)\right] \\
& \times\left[1-F\left(\left\{z_{i}^{\prime}(x) \wedge y_{i-1}^{\prime}(x)\right\} \vee y_{i+1}^{\prime}(x) \mid w\right)\right] g(w) d w \\
& \left.+\int_{w_{2}^{i}}^{w_{1}^{i+1}} \phi_{i}^{+}\left(z_{i}^{\prime}(x), w\right)\left[1-F\left(\phi_{i}^{+}\left(z_{i}^{\prime}(x), w\right) \mid w\right)\right] g(w) d w\right\} d x .
\end{aligned}
$$

This is a pointwise maximization problem, and the optimal $z_{i}^{\prime}$ is constant.

We can now characterize the pricing function for $w>\tau(1)$.

Lemma C6. For any $T$ satisfying $(V F)$ and $\left(W F^{\prime}\right)$, there exists $T^{*}$ satisfying $(V F)$ and $\left(W F^{\prime}\right)$ that yields weakly higher expected revenue, where $T^{*}(x, w)=\int_{0}^{x}\left\{\tau^{\prime}(s) \wedge \bar{m}_{r(1)}(w)\right\} d s$ for $w>\tau(1)$.

Proof. By Lemmas $\mathrm{C} 1$ and $\mathrm{C} 5$, we can restrict attention to a pricing function $T^{*}$ that satisfies $T^{*}(x, w)=\tau(x)$ for $w \leqslant \tau(1)$ and $T^{*}(x, w)=$ $\int_{0}^{x}\left\{\tau^{\prime}(s) \wedge p(w)\right\} d s$ for $w>\tau(1)$, for some $p:(\tau(1), \bar{w}] \rightarrow R$. Now fix $x \in[0,1]$, and let $p^{*}:[\tau(1), \bar{w}] \rightarrow \mathfrak{R}_{+}$solve

$$
\max _{p} \int_{\tau(1)}^{\bar{w}}\left\{\tau^{\prime}(x) \wedge p(w)\right\}\left[1-F\left(\tau^{\prime}(x) \wedge p(w) \mid w\right)\right] g(w) d w
$$

s.t. $p$ is nonincreasing.

Note that $T^{*}$ satisfying $T^{*}(x, w)=\tau(x)$ for $w \leqslant \tau(1)$, and $T^{*}(x, w)=$ $\int_{0}^{x}\left\{\tau^{\prime}(s) \wedge p^{*}(w)\right\} d s$ for $w>\tau(1)$, satisfies $(V F)$ and $\left(W F^{\prime}\right)$. Moreover, $T^{*}$ yields weakly higher revenue than $T$.

It now suffices to show that $\bar{m}_{\tau(1)}$ solves the above program for all $x \in[0,1]$. To that end, first let $\hat{W}(p) \equiv \inf \left\{w \in[\tau(1), \bar{w}] \mid p^{*}(w)<p\right\}$, and let $\hat{w}(p) \equiv \inf \left\{w \in[\tau(1), \bar{w}] \mid \bar{m}_{\tau(1)}(w)<p\right\}$. (The infimum is taken to be $\bar{w}$ if the relevant set is empty.) Note that $\bar{m}_{\tau(1)}(w)$ is strictly decreasing in $\left[\hat{w}\left(\tau^{\prime}(x)\right), \hat{w}\left(\tau^{\prime}(x)\right)+\varepsilon\right)$, for some $\varepsilon>0$; otherwise, $\hat{w}\left(\tau^{\prime}(x)\right)=\tau(1)$ or 
$\hat{w}\left(\tau^{\prime}(x)\right)=\bar{w}$. Then, by Lemma C3, $\bar{m}_{\tau(1)}(w)=\bar{m}_{\hat{w}\left(\tau^{\prime}(x)\right)}(w)$ for all $w>\hat{w}\left(\tau^{\prime}(x)\right)$. Using the symmetric argument, $p^{*}(w)=\bar{m}_{\hat{W}\left(\tau^{\prime}(x)\right)}(w)$ for all $w>\hat{W}\left(\tau^{\prime}(x)\right)$.

There are two cases to consider. Suppose first that $\hat{w}\left(\tau^{\prime}(x)\right) \leqslant \hat{W}\left(\tau^{\prime}(x)\right)$. Then, $\bar{m}_{\tau(1)}$ clearly solves the problem since $\bar{m}_{\tau(1)}(w)$ weakly dominates $p^{*}(w)$ for $w>\hat{w}\left(\tau^{\prime}(x)\right)$, whereas the two functions give the same value of the integrand (i.e., $\left.\tau^{\prime}(x)\right)$ for $w<\hat{w}\left(\tau^{\prime}(x)\right)$. Now suppose that $\hat{W}\left(\tau^{\prime}(x)\right)<$ $\hat{w}\left(\tau^{\prime}(x)\right)$. Consider the function $\psi:[\tau(1) \wedge \underline{w}, \bar{w}] \rightarrow \mathfrak{R}$ such that $\psi(w)=$ $\bar{m}_{\tau(1)}(w)$ for $w \in\left[\tau(1) \wedge \underline{w}, \hat{W}\left(\tau^{\prime}(x)\right)\right]$ and $\psi(w)=\bar{m}_{\hat{W}\left(\tau^{\prime}(x)\right)}(w)$ for $w>$ $\hat{W}\left(\tau^{\prime}(x)\right)$. Clearly, $\psi$ is nonincreasing since $\hat{W}\left(\tau^{\prime}(x)\right)<\hat{w}\left(\tau^{\prime}(x)\right)$, and it strictly dominates $\bar{m}_{\tau(1)}$, which contradicts the fact that $\bar{m}_{\tau(1)}$ solves the program $\left[\mathrm{M}_{\tau(1)}\right]$. We conclude that $\bar{m}_{\tau(1)}$ solves the above program for each $x \in[0,1]$.

\section{APPENDIX D}

\section{Proof of Theorem 2}

In order to show existence of $\tau$ that solves [S], we consider a slightly relaxed program:

$$
\max _{\tau:[0,1] \rightarrow \mathfrak{R}_{+}} \int_{0}^{1} \phi\left(x, \tau(x), \tau^{\prime}(x)\right) d x
$$

subject to

$$
\begin{array}{r}
\tau:[0,1] \rightarrow \mathfrak{R}_{+} \quad \text { is a continuous function with } \\
\tau(0)=0, \quad 0 \leqslant \tau^{\prime} \leqslant \bar{v} \text { and } \tau^{\prime} \leqslant \tau^{\prime}(1),
\end{array}
$$

where

$$
\begin{aligned}
\phi(x, \tau, u) \equiv & \int_{\tau}^{\tau(1)} u[1-F(u \mid w)] g(w) d w \\
& +\int_{\tau(1)}^{\bar{w}}\left(u \wedge \bar{m}_{\tau(1)}(w)\right)\left[1-F\left(u \wedge \bar{m}_{\tau(1)}(w) \mid w\right)\right] g(w) d w
\end{aligned}
$$

The condition $\tau^{\prime} \leqslant \tau^{\prime}(1)$ is weaker than the convexity constraint, so [ $\left.S 1\right]$ is a relaxed version of $[S]$.

We first establish existence of a solution to [S1]. This is accomplished by invoking Theorem 2.1 of Fleming and Rishel [4, p. 63]. To see that the conditions of the theorem are met, note first that the feasible set of functions satisfying the constraints is nonempty (condition (a) of Theorem 2.1), 
which can be checked by observing that $\tau(x)=0$ satisfies all of the constraints. Next, observe that $\tau^{\prime}$ lies in a compact set $[0, \bar{v}]$ (condition (b)). Third, the set of terminal values is in a compact set, $[0, \bar{v}]$, and $\phi$ varies continuously with the terminal value (condition (c)). Finally, $\phi(x, \tau, u)$ is continuous in $u$, so the image of $[0, \bar{v}]$ under $\phi(x, \tau, \cdot)$ is convex (condition (d)). It therefore follows that a solution to $[S 1]$ (in the space of Lebesque integrable functions) exists.

Below, we characterize the necessary conditions for the solution to [ $S 1]$ and show that they imply that the solution to $[S 1]$ satisfies all the constraints of $[S]$, which will prove that the solution to $[S 1]$ is also a solution to $[S]$.

Consider the Lagrangean associated with $[S 1]$ :

$$
J(x, \tau, u) \equiv \phi(x, \tau, u)+\lambda u+\lambda_{1} u+\lambda_{2}(\bar{v}-u),
$$

where $\lambda$ is the costate variable, and $\lambda_{1}$ and $\lambda_{2}$ are the multipliers associated with the control bounds. The Hamiltonian necessary conditions are:

$$
\begin{aligned}
& \tau^{\prime}=u, \\
& \lambda^{\prime}=-\frac{\partial \phi}{\partial \tau} .
\end{aligned}
$$

Fixing $x \in[0,1]$, condition (D3) implies that

$$
\begin{aligned}
\lambda(x) & =\lambda(1)+\int_{x}^{1} \frac{\partial \phi(s, \tau, u)}{\partial \tau} d s \\
& =\lambda(1)-\int_{x}^{1} u(s)[1-F(u(s) \mid \tau(s))] g(\tau(s)) d s .
\end{aligned}
$$

Substituting (D4) into (D1) and differentiating, the Pontryagin maximum principle yields the first-order necessary condition:

$$
\begin{aligned}
0=J_{u}= & \int_{\tau(x)}^{\hat{w}(u(x))} H(u(x) \mid w) g(w) d w \\
& -\int_{x}^{1} u(s)[1-F(u(s) \mid \tau(s))] g(\tau(s)) d s+\lambda(1)+\lambda_{1}(x)-\lambda_{2}(x) \\
= & \int_{\tau(1)}^{\hat{w}(u(x))} H(u(x) \mid w) g(w) d w \\
& -\int_{x}^{1} u(s)\{-H(u(x) \mid \tau(s))+[1-F(u(s) \mid \tau(s))]\} g(\tau(s)) d s \\
& +\lambda(1)+\lambda_{1}(x)-\lambda_{2}(x) .
\end{aligned}
$$


The complementary slackness conditions are:

$$
\begin{array}{lll}
u(x) \geqslant 0, & \lambda_{1}(x) \geqslant 0, & \lambda_{1}(x) u(x)=0 ; \\
u(x) \leqslant \bar{v}, & \lambda_{2}(x) \geqslant 0, & \lambda_{2}(x)(\bar{v}-u(x))=0,
\end{array}
$$

for $u(x) \neq \underline{v}$, where we use the fact that $u=\bar{m}_{\tau(1)}(\hat{w}(u))$ whenever $\hat{w}(u)$ varies with $u .(H(u \mid w)$ jumps down at $u=\underline{v}$ unless $\underline{v} f(\underline{v} \mid w)=0$.) Finally, since $\tau$ is free at $x=1$,

$$
\begin{aligned}
\lambda(1)= & \int_{0}^{1} \frac{\partial \phi}{\partial \tau(1)} d x \\
= & \int_{0}^{1} g(\tau(1))\{u(x)[1-F(u(x) \mid \tau(1))] \\
& -(u(x) \wedge \mu(\tau(1)))[1-F(u(x) \wedge \mu(\tau(1)) \mid \tau(1))]\} d x \\
= & g(\tau(1)) \int_{\{u(x)>\mu(\tau(1))\}}\{u(x)[1-F(u(x) \mid \tau(1))] \\
& -\mu(\tau(1))[1-F(\mu(\tau(1)) \mid \tau(1))]\} d x
\end{aligned}
$$

By the above existence result, there exist functions $\tau$ and $u$ that satisfy (D2), (D5), (D6) and (D7). Let $\tau^{*}$ and $u^{*}$ denote the solutions, respectively. We make the following observations about the solution.

Lemma D1. At the solution to $[S 1], \lambda(1)=0$. Furthermore, if $\tau^{*}(1) \in$ $[\underline{w}, \bar{w}], u^{*}(1) \leqslant \mu(\tau *(1))$.

Proof. First note from (D7) that $\lambda(1) \leqslant 0$ because the integrand of (D7),

$$
u(x)[1-F(u(x) \mid \tau(1))]-\mu(\tau(1))[1-F(\mu(\tau(1)) \mid \tau(1))],
$$

is negative whenever $u(x)>\mu(\tau(1))$. This follows from Assumption 1 (concavity of $u(x)[1-F(u(x) \mid \tau(1))]$ in $u(x))$ since $\mu(\tau(1)) \geqslant m(\tau(1))$. We now show that $\lambda(1)=0$. Clearly, $\lambda(1)=0$ if $\tau^{*}(1)>\bar{w}$ or if $\tau^{*}(1)<\underline{w}$. Thus, it suffices to show that $\lambda(1)=0$ for $\tau^{*}(1) \in[\underline{w}, \bar{w}]$. Suppose, to the contrary, that $\lambda(1)<0$. Since $u^{*} \leqslant u^{*}(1)$ (by the last condition of $[S 1]$ ), we must then have $u^{*}(1)>\mu\left(\tau^{*}(1)\right)$. It follows that $\hat{w}\left(u^{*}(1)\right)=\tau^{*}(1)$. Moreover, since $\mu>0, u^{*}(1)>0$, so $\lambda_{1}(1)=0$ by (D6). Therefore, at $x=1, J_{u}=$ $\lambda(1)-\lambda_{2}(1)<0$. This contradicts (D5), so we conclude that $\lambda(1)=0$, which in turn implies that $u^{*}(1) \leqslant \mu\left(\tau^{*}(1)\right)$.

Lemma D2. If $\tau^{*}(1) \in[\underline{w}, \bar{w})$, then $u^{*}(1)=\mu\left(\tau^{*}(1)\right)$; if $\tau^{*}(1)<\underline{w}$, then $u^{*}(1) \geqslant \mu\left(\tau^{*}(1)\right)=\mu(\underline{w})$. 
Proof. Given Lemma D1, it suffices to show that $u^{*}(1) \geqslant \mu\left(\tau^{*}(1)\right)$ if $\tau^{*}(1)<\bar{w}$. Suppose, to the contrary, that $u^{*}(1)<\mu\left(\tau^{*}(1)\right)$. Then, since $\mu<\bar{v}, \lambda_{2}(1)=0$, by (D6). Therefore, at $x=1$,

$$
J_{u}=\int_{\tau^{*}(1)}^{\hat{w}\left(u^{*}(1)\right)} H\left(u^{*}(1) \mid w\right) g(w) d w+\lambda(1)>0,
$$

by Lemma C2, since $u^{*}(1)<\bar{m}_{\tau^{*}(1)}(w)$ for $w \in\left[\tau^{*}(1), \hat{w}\left(u^{*}(1)\right)\right)$ and $\tau^{*}(1)$ $<\hat{w}\left(u^{*}(1)\right)$. This contradicts (D5), so $u^{*}(1) \geqslant \mu\left(\tau^{*}(1)\right)$ for $\tau^{*}(1)<\bar{w}$.

Lemma D3. At the solution, $\lambda_{2}(x)=0$ for all $x \in[0,1]$.

Proof. Suppose, to the contrary, that $\lambda_{2}(x)>0$. Then, by (D6), $u^{*}(x)=\bar{v}$ and $\lambda(1)=0$. It follows that $H\left(u^{*}(x) \mid w\right)=-\bar{v} f(\bar{v} \mid w)$, so $J_{u}<0$, which contradicts (D5). Therefore, $\lambda_{2}(x)=0$ for all $x \in[0,1]$.

Lemma D4. Without loss of generality, $u^{*}(x) \geqslant \underline{v}$ for all $x \in[0,1]$.

Proof. Fix $x \in[0,1]$ and suppose that $u(x)<\underline{v}$, which implies that $H(u(x) \mid w)=1$ for all $w$. Since $\bar{m}_{z}(w) \geqslant \underline{v}$ for all $z$ and for $w \in[z \wedge \underline{w}, \bar{w}]$, we have $\hat{w}(u(x))=\bar{w}$. It then follows that $J_{u} \geqslant 0$. (In the second line of (D5), the first integral term is nonnegative since $H(u(x) \mid w)=1$ when $u(x)<\underline{v}$. The second integral term is also nonnegative since $-H(u(x) \mid \tau(s))$ $+[1-F(u(s) \mid \tau(s))]=-1+[1-F(u(s) \mid \tau(s))] \leqslant 0$.) Therefore, $u^{*}(x) \geqslant \underline{v}$ is optimal.

Lemma D5. Suppose that $\tau^{*}(1)<\bar{w}$. Then, a solution to $(D 1)$ satisfies (2) whenever $u^{*}(x)>0$.

Proof. By Lemma D3, $\lambda_{2}=0$. Also, whenever $u^{*}(x)>0, \lambda_{1}(x)=0$ by (D6). Hence, (D5) must hold with $\lambda_{1}(x)=\lambda_{2}(x)=0$ whenever $u^{*}(x)>0$. If $\tau^{*}(1)<\bar{w}$, then $\hat{w}\left(u^{*}(1)\right)=\bar{w}>\tau^{*}(1)$. By the implicit function theorem, the derivative of $u^{*}$ exists whenever $u^{*}(x)>0$, and it is characterized by (2), which is obtained by totally differentiating both sides of (D5) with respect to $x$ (using $\lambda_{1}(x)=\lambda_{2}(x)=0$ ). In the process of differentiating, we use the fact that the derivative of $J_{u}$ with respect to $u$, through $\hat{w}(u)$, vanishes. This point is not trivial, since $\hat{w}$ may jump down. We prove the result as follows. First, since $\hat{w}$ is monotonically decreasing, it can only jump down at countably many points. In particular, the left-hand derivative of $\hat{w}$ is well-defined. (Since $\hat{w}(u) \equiv \inf \left\{w \in[\tau(1), \bar{w}] \mid \bar{m}_{\tau(1)}(w)<u\right\}, \hat{w}\left(u^{\prime}\right)$ is strictly decreasing in $u^{\prime}$ for $u^{\prime} \in(u-\varepsilon, u]$ for some $\varepsilon>0$. Therefore, the left-hand derivative exists, by the implicit function theorem.) Let $\hat{w}^{\prime}$ denote the left-hand derivative. Next, let $\tilde{w}(u) \equiv \sup \left\{w \in[\tau(1), \bar{w}] \mid \bar{m}_{\tau(1)}(w)>u\right\}$ if the set is non-empty; otherwise $\tilde{w}(u) \equiv \tau(1)$. Then, for the same reason, the right-hand derivative of $\tilde{w}$ exists, and we denote it by $\tilde{w}^{\prime}$. 
The left-hand derivative of $J_{u}$ with respect to $u$, through $\hat{w}(u)$, is

$$
\lim _{u^{\prime} \uparrow u} \frac{\int_{\hat{w}(u)}^{\hat{w}\left(u^{\prime}\right)} H(u \mid w) g(w) d w}{u^{\prime}-u}=\hat{w}^{\prime}(u) H(u \mid \hat{w}(u)) g(\hat{w}(u))=0,
$$

since $u=\bar{m}_{\tau(1)}(\hat{w}(u))=m(\hat{w}(u))$ whenever $\hat{w}$ is strictly decreasing.

Now consider the right-hand derivative of $J_{u}$ with respect to $u$, through $\hat{w}(u)$ :

$$
\begin{aligned}
\lim _{u^{\prime} \downarrow u} & \frac{\int_{\hat{w}(u)}^{\hat{w}\left(u^{\prime}\right)} H(u \mid w) g(w) d w}{u^{\prime}-u} \\
& =\lim _{u^{\prime} \downarrow u} \frac{\int_{\tilde{w}(u)}^{\tilde{w}(u)} H(u \mid w) g(w) d w+\int_{\tilde{w}(u)}^{\hat{w}\left(u^{\prime}\right)} H(u \mid w) g(w) d w}{u^{\prime}-u} \\
& =0+\lim _{u^{\prime} \downarrow u} \frac{\int_{\tilde{w}(u)}^{\hat{w}\left(u^{\prime}\right)} H(u \mid w) g(w) d w}{u^{\prime}-u} \\
& =\tilde{w}^{\prime}(u) H(u \mid \tilde{w}(u)) g(\tilde{w}(u))=0,
\end{aligned}
$$

where the second equality holds by Lemma $\mathrm{C} 2$ and the last equality holds since $u=\bar{m}_{\tau(1)}(\tilde{w}(u))=m(\tilde{w}(u))$ whenever $\tilde{w}$ is strictly decreasing.

We now show that the solution to the relaxed program satisfies all of the constraints of $[\mathrm{S}]$.

\section{Lemma D6. The solution to $[S 1], \tau^{*}$, is convex and continuous.}

Proof. By Assumption 1, $J(x, \tau, u)$ is strictly concave in $u \in[\underline{v}, \bar{v}]$, so $J^{*}(x, u) \equiv J\left(x, \tau^{*}(x), u\right)$ attains a unique maximum at $u=u^{*}(x)$. This, together with the fact that $\phi$ is $C^{1}$ for $u \in(\underline{v}, \bar{v})$, implies that $u^{*}$ is continuous (see Theorem 6.1 of Fleming and Rishel [4, p. 75]). Continuity of $u^{*}$ immediately implies that $\tau^{*}$ is continuous. Continuity of $u^{*}$ also implies convexity of $\tau^{*}$. To see this, observe from (1) that $\tau^{*}$ is convex for $\tau^{*}(x) \in$ $(\underline{w}, \bar{w})$, and it is linear elsewhere. Combined with the continuity of $u^{*}$, this means that $u^{*}$ can never decrease, implying that $\tau^{*}$ is convex.

By Lemma D6, the solution to the relaxed program [S1], $\tau^{*}$, satisfies all of the constraints of [S]. Hence, $\tau^{*}$ must solve [S]. Lemma 4 then implies that there exists $T^{*}$ with the stated properties that implements the optimal selling mechanism.

\section{REFERENCES}

1. M. Armstrong, Multiproduct nonlinear pricing, Econometrica 64 (1996), 51-75.

2. C. F. Bergsten, K. A. Elliott, J. J. Schott, and W. E. Takacs, "Auction Quotas and United States Trade Policy," Institute for International Economics, Washington, DC, 1987. 
3. R. Deneckere and R. P. McAfee, Damaged goods, J. Econ. Manage. Strategy 5 (1996), 149-174.

4. W. H. Fleming and R. M. Rishel, "Deterministic and Stochastic Optimal Control," Springer-Verlag, New York, 1975.

5. F. Gul, H. Sonnenschein, and R. Wilson, Foundations of dynamic monopoly and the Coase conjecture, J. Econ. Theory 39 (1986), 155-190.

6. M. Harris and A. Raviv, A theory of monopoly pricing schemes with demand uncertainty, Amer. Econ. Rev. 71 (1981), 347-365.

7. K. Hendricks and R. Porter, Joint bidding in federal OCS auctions, Amer. Econ. Rev. Papers Proc. 82 (1992), 506-511.

8. P. Jehiel, B. Moldovanu, and E. Stachetti, Multidimensional mechanism design for auctions with externalities, mimeo, University of Michigan, 1994.

9. M. L. Katz, Nonuniform pricing with unobservable numbers of purchases, Rev. Econ. Stud. LI (1984), 46-70.

10. J.-J. Laffont, E. Maskin, and J.-C. Rochet, Optimal nonlinear pricing with two-dimensional characteristics, in "Information, Incentives, and Economic Mechanisms" (T. Groves, R. Radner, and S. Reiter, Eds.), University of Minnesota Press, Minneapolis, 1987.

11. E. Maskin and J. Riley, Monopoly with incomplete information, Rand J. Econ. 15 (1984), 171-196.

12. R. P. McAfee and J. McMillan, Multidimensional incentive compatibility and mechanism design, J. Econ. Theory 46 (1988), 335-354.

13. P. Milgrom and C. Shannon, Monotone comparative statics, Econometrica 62 (1994), $157-180$.

14. M. Mussa and S. Rosen, Monopoly and product quality, J. Econ. Theory 18 (1978), 301-317.

15. J. Riley and R. Zeckhauser, Optimal selling strategies: when to haggle, when to hold firm, Quart. J. Econ. (1983), 267-289.

16. J.-C. Rochet and P. Choné, Ironing, sweeping and multidimensional screening, Econometrica 66 (1998), 783-826.

17. R. T. Rockafellar, "Convex Analysis," Princeton University Press, Princeton, 1970.

18. D. Salant, Up in the air: GTE's experience in the MTA auction for personal communication services licenses, J. Econ. Manage. Strategy 6 (1997), 549-572.

19. A. Sen, On seller financing of consumer durables, J. Econ. Manage. Strategy 7 (1998), 435-460.

20. N. Stokey, Intertemporal price discrimination, Quart. J. Econ. 93 (1979), 355-371.

21. C. Wilson, On the optimal pricing policy of a monopolist, J. Polit. Econ. 96 (1988), 164-176.

22. R. Wilson, Efficient and competitive rationing, Econometrica 57 (1989), 1-40. 